\title{
COHOMOLOGY AND BASE CHANGE FOR ALGEBRAIC STACKS
}

\author{
JACK HALL
}

\begin{abstract}
We prove that cohomology and base change holds for algebraic stacks, generalizing work of Brochard in the tame case. We also show that Hom-spaces on algebraic stacks are represented by abelian cones, generalizing results of Grothendieck, Brochard, Olsson, Lieblich, and Roth-Starr. To accomplish all of this, we prove that a wide class of Ext-functors in algebraic geometry are coherent (in the sense of M. Auslander).
\end{abstract}

\section{INTRODUCTION}

Our first main result is the following version of cohomology and base change.

Theorem A. Fix a morphism of locally noetherian algebraic stacks $f: X \rightarrow S$ which is separated and locally of finite type, $\mathcal{M}^{\bullet} \in \mathrm{D}_{\text {Coh }}^{-}(X)$, and $\mathcal{N} \in \operatorname{Coh}(X)$ which is properly supported and flat over $S$. For each integer $q \geq 0$ and morphism of noetherian algebraic stacks $g: T \rightarrow S$, there is a natural base change morphism:

$$
b^{q}(T): g^{*} \mathcal{E} x t^{q}(f ; \mathcal{M} \cdot, \mathcal{N}) \rightarrow \mathcal{E} x t^{q}\left(f_{T} ; \mathrm{L}\left(g_{X}\right)_{\mathrm{Q}}^{*} \mathcal{M} \bullet, g_{X}^{*} \mathcal{N}\right),
$$

where $f_{T}: X_{T} \rightarrow T$ denotes the pullback of $f$ by $g$ and $g_{X}: X_{T} \rightarrow X$ denotes the pullback of $g$ by $f$. Now fix $s \in|S|$ such that $b^{q}(s)$ is surjective.

(1) Then, there exists an open neighbourhood $U \subseteq S$ of $s \in|S|$ such that for any $g: T \rightarrow S$ factoring through $U$, the map $b^{q}(T)$ is an isomorphism.

(2) The following are equivalent:

(a) $b^{q+1}(s)$ is surjective,

(b) the coherent $\mathcal{O}_{S}$-module $\mathcal{E} x t^{q+1}(f ; \mathcal{M} \cdot, \mathcal{N})$ is free at $s$.

In the proof of Theorem $\mathrm{A}$ for projective morphisms [EGA, III.7.7.5], the essential point was to corepresent the relevant functors by bounded above complexes of coherent sheaves on $S$. Thus the following notion was indispensible: for a noetherian scheme $S$, a functor $\mathbf{Q C o h}(S) \rightarrow \mathbf{Q C o h}(S)$ is corepresentable by a complex if it is of the form $\mathcal{J} \mapsto \mathcal{H}^{0}\left(\mathrm{R} \mathcal{H} \operatorname{lom}_{\mathcal{O}_{S}}\left(Q^{\bullet}, \mathcal{J}\right)\right)$ where $Q^{\bullet} \in \mathrm{D}_{\text {Coh }}^{-}(S)$.

Brochard noticed [Bro12, App. A], however, that corepresenting the cohomology functors of a non-tame stack by complexes is quite subtle - the problem is that these stacks tend to have infinite cohomological dimension. In particular, the "Mumford Lemma" Mum70, Lem. 5.1] is no longer applicable. Our next main result shows that this problem can be circumvented when one has duality.

Theorem B. Fix a noetherian algebraic stack $S$ which admits a dualizing complex $\mathcal{K}^{\bullet}$, and a morphism of algebraic stacks $f: X \rightarrow S$ which is proper. Let $\mathcal{N}^{\bullet} \in$

Date: March 8, 2013.

2010 Mathematics Subject Classification. Primary 14F05; Secondary 13D09, 14D20, 14D23. 
$\mathrm{D}_{\mathrm{Coh}}^{-}(X)$ and $\mathcal{N}^{\bullet} \in \mathrm{D}_{\mathrm{Coh}}^{b}(X)$. If $\mathcal{N}^{\bullet}$ has finite tor-dimension over $S$, then there exists a quasi-isomorphism:

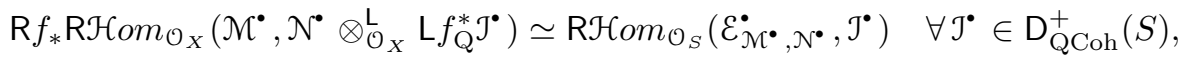

natural in $\mathrm{J}^{\bullet}$, where:

$$
\mathcal{E}_{\mathfrak{\mathcal { N }}^{\bullet}, \mathfrak{N}^{\bullet}} \simeq \mathrm{RH}_{\mathcal{H} o m_{\mathcal{O}_{S}}}\left(\mathrm{R} f_{*} \mathrm{R} \mathcal{H} \operatorname{Hom}_{\mathcal{O}_{X}}\left(\mathcal{M}^{\bullet}, \mathcal{N}^{\bullet} \otimes_{\mathcal{O}_{X}}^{\mathrm{L}} \mathrm{L} f_{\mathrm{Q}}^{*} \mathcal{K}^{\bullet}\right), \mathcal{K}^{\bullet}\right) \in \mathrm{D}_{\mathrm{Coh}}^{-}(S) .
$$

In addition, if $\mathcal{N}^{\bullet} \simeq \mathcal{N}[0]$, where $\mathcal{N} \in \operatorname{Coh}(X)$ is flat over $S$, then the formation of $\varepsilon_{\mathcal{N} \cdot, \mathfrak{N} \cdot}$ is compatible with base change.

Since any separated scheme of finite type over a Gorenstein ring (e.g. Spec $\mathbb{Z}$ or a field) and the spectrum of any maximal-adically complete noetherian local ring admits a dualizing complex. Thus, Theorem B covers most cases encountered in practice (in particular, it generalizes [BF97, Lem. 6.1]).

We wish to point out, however, that the collection of functors $\mathrm{QCoh}(S) \rightarrow$ $\mathbf{Q C o h}(S)$ which are corepresentable by a complex is poorly behaved. Indeed, it is not even closed under direct summands Har98, Prop. 4.6 \& Ex. 5.5].

The following generalization of functors which are corepresentable by a complex was considered by M. Auslander Aus66 in order to correct such deficiencies. For an affine (not necessarily noetherian) scheme $S$, a functor $F: \mathbf{Q C o h}(S) \rightarrow \mathbf{A b}$ is coherent if there exists a morphism of quasicoherent $\mathcal{O}_{S}$-modules $\mathcal{K}_{1} \rightarrow \mathcal{K}_{2}$, such that for all $\mathcal{J} \in \mathbf{Q C o h}(S)$, there is a natural isomorphism of abelian groups:

$$
F(\mathcal{J}) \cong \operatorname{coker}\left(\operatorname{Hom}_{\mathcal{O}_{S}}\left(\mathcal{K}_{2}, \mathcal{J}\right) \rightarrow \operatorname{Hom}_{\mathcal{O}_{S}}\left(\mathcal{K}_{1}, \mathcal{J}\right)\right) .
$$

Note that if $S$ is noetherian, the coherent functors of R. Hartshorne Har98 are precisely those coherent functors of M. Auslander Aus66 which preserve direct limits.

In any case, the collection of coherent functors is very well-behaved: it is an abelian category which is closed under extensions and inverse limits - precisely the sort of properties that are convenient to have at one's disposal when performing induction arguments. Thus, using Theorem B we can prove the following Theorem.

Theorem C. Fix an affine scheme $S$, a morphism of algebraic stacks $f: X \rightarrow S$ which is separated and locally of finite presentation, $\mathcal{M}^{\bullet} \in \mathrm{D}_{\mathrm{QCoh}}(X)$, and $\mathcal{N} \in$ $\mathrm{QCoh}(X)$, with $\mathcal{N}$ of finite presentation, properly supported and flat over $S$. Then, the functor:

$$
\operatorname{Hom}_{\mathcal{O}_{X}}\left(\mathcal{M} \cdot, \mathcal{N} \otimes_{\mathcal{O}_{X}}^{\mathrm{L}} \mathrm{L} f_{\mathbf{Q}}^{*}(-)\right): \mathbf{Q C o h}(S) \rightarrow \mathbf{A b}
$$

is coherent.

We wish to emphasize that Theorem C eliminates from Theorem B the finiteness hypotheses on $S$ (i.e. $S$ is permitted to be non-noetherian) and on $\mathcal{M}^{*}$ (i.e. $\mathcal{M}^{\bullet}$ can be unbounded with quasicoherent cohomology). Moreover, the employment of Auslander's definition of coherent functors is essential for the proof and truth of Theorem $\mathrm{C}$ If $S$ is noetherian and $\mathcal{M}^{*} \in \mathrm{D}_{\mathrm{Coh}}^{-}(X)$, then Theorem $\mathrm{C}$ implies that the functor is coherent in the sense of Hartshorne (see Lemma 1.1).

Theorem $\mathrm{A}$ is proved by combining a clever result of A. Ogus and G. Bergman OB72, Cor. 5.1], Theorem C and some general vanishing results for coherent functors. An interesting application of Theorem $\mathrm{C}$ is the following: given a scheme 
$S$, an algebraic $S$-stack $X$, and $\mathcal{M}, \mathcal{N} \in \mathbf{Q C o h}(X)$, we define the $S$-presheaf $\underline{\operatorname{Hom}}_{\mathcal{O}_{X} / S}(\mathcal{M}, \mathcal{N})$ as follows:

$$
\underline{\operatorname{Hom}}_{\mathcal{O}_{X} / S}(\mathcal{M}, \mathcal{N})[T \stackrel{\tau}{\rightarrow} S]=\operatorname{Hom}_{\mathcal{O}_{X_{T}}}\left(\tau_{X}^{*} \mathcal{M}, \tau_{X}^{*} \mathcal{N}\right)
$$

where $\tau_{X}: X \times_{S} T \rightarrow X$ is the projection. Then, we prove

Theorem D. Fix a scheme $S$ and a morphism of algebraic stacks $f: X \rightarrow S$, which is separated and locally of finite presentation. Let $\mathcal{N}, \mathcal{N} \in \operatorname{QCoh}(X)$, with $\mathcal{N}$ of finite presentation, flat over $S$, with support proper over $S$. Then, $\underline{\operatorname{Hom}}_{\mathcal{O}_{X} / S}(\mathcal{M}, \mathcal{N})$ is representable by an abelian cone over $S$ (which is, in particular, affine over $S$ ). If $\mathcal{M}$ is of finite presentation, then $\underline{\operatorname{Hom}}_{\mathcal{O}_{X} / S}(\mathcal{M}, \mathcal{N})$ is finitely presented over $S$.

We wish to emphasize that Theorem $\mathrm{D}$ is completely elementary once Theorem C is known. Using different techniques to that employed in Theorem C, we prove a coherence result when nothing is assumed to be flat (at the expense of making the diagonal finite).

Theorem E. Fix an affine and noetherian scheme $S$, a morphism of algebraic stacks $f: X \rightarrow S$ which is locally of finite type with finite diagonal, $\mathcal{M} \cdot \in \mathrm{D}_{\mathrm{QCoh}}(X)$, and $\mathcal{N}^{\bullet} \in \mathrm{D}_{\text {Coh }}^{b}(X)$. If $\mathcal{N}^{\bullet}$ has properly supported cohomology sheaves over $S$, then the functor:

$$
\operatorname{Hom}_{\mathcal{O}_{X}}\left(\mathcal{M} \cdot, \mathcal{N}^{\bullet} \otimes_{\mathcal{O}_{X}}^{\mathrm{L}} \mathrm{L} f_{\mathrm{Q}}^{*}(-)\right): \mathbf{Q C o h}(S) \rightarrow \mathbf{A b}
$$

is coherent.

We wish to emphasize that Theorem $\mathrm{E}$ is independent of Theorem $\mathrm{B}$

Relation with other work. In Hal12, coherent functors featured prominently in a criteria for algebraicity of stacks. Thus Theorem $\mathrm{C}$ can be used to show that certain stacks are algebraic [op. cit., $\S \S 8-9]$. Theorem [D can also be used to show that many algebraic stacks of interest have affine diagonals [Hal12, $\S \S 8-9]$ generalizing and simplifying the existing work of M. Olsson Ols06, Prop. 5.10], M. Lieblich [Lie06, Prop. 2.3], and M. Roth and J. Starr [RS09, Thm. 2.1]. In HR12b, Theorem [D is combined with the absolute approximation results of D. Rydh Ryd09 to show that Hilbert stacks and Quot spaces exist without finiteness assumptions.

Results of A. Grothendieck [EGA, III.7.7.8-9], A. Altman and S. Kleiman [AK80, 1.1], and S. Brochard [Bro12, Prop. A.4.3] demonstrate that Theorem Aholds when $f$ is proper, tame, and flat, and $\mathcal{M}$ is flat over $S$ as well as being the cokernel of a map vector bundles. For example, if $f$ is projective, or more generally, $X$ is tame and has the resolution property, Theorem $\mathrm{A}$ is known to hold for any coherent sheaf $\mathcal{M}$ which is flat over $S$.

After completing this paper we also located in the literature two very nice papers of $\mathrm{H}$. Flenner addressing similar results for analytic spaces. In particular, if $S$ is excellent and of finite Krull dimension, then Theorem $\mathrm{A}$ follows from the results of [Fle81, §7] and Theorems B and D are the main results of [Fle82] (this is in the analytic category, however, thus $\mathcal{M}$ is assumed to be coherent in Theorem (D). Without further assumptions on $f$ and $\mathcal{M}^{\bullet}$ (e.g. $\mathcal{M}^{\bullet}$ and $X$ are flat over $S$ when $q \geq 1$ [K80, $\S 1]$ ) we cannot see how Theorem $\mathrm{A}$ can be easily reduced to the case where $S$ meets Flenner's hypotheses. In fact, we use coherent functors to accomplish this descent, which is effectively the content of Theorem C. This has no counterpart in the analytic category where everything is excellent, coherent, and 
admits a dualizing complex. We do not believe that TheoremE $\mathrm{E}$ has been considered previously.

Assumptions, conventions, and notations. For a scheme $T$, denote by $|T|$ the underlying topological space (with the Zariski topology) and $\mathcal{O}_{T}$ the (Zariski) sheaf of rings on $|T|$. For $t \in|T|$, let $\kappa(t)$ denote the residue field. Denote by $\mathbf{Q C o h}(T)$ (resp. $\mathbf{C o h}(T)$ ) the abelian category of quasicoherent (resp. coherent) sheaves on the scheme $T$. Let $\mathbf{S c h} / T$ denote the category of schemes over $T$. The big étale site over $T$ will be denoted by $(\mathbf{S c h} / T)_{\text {Ét }}$.

For a ring $A$ and an $A$-module $M$, denote the quasicoherent $\mathcal{O}_{\mathrm{Spec} A} A_{\text {-module }}$ associated to $M$ by $\widetilde{M}$. Denote the abelian category of all (resp. coherent) $A$ $\operatorname{modules}$ by $\operatorname{Mod}(A)(\operatorname{resp} . \mathbf{C o h}(A))$.

We will assume throughout that all schemes, algebraic spaces, and algebraic stacks have quasicompact and quasiseparated diagonals.

Acknowledgements. I would like to thank R. Ile and D. Rydh for several interesting and supportive discussions.

\section{Contents}

Introduction

1. Derived categories of sheaves on algebraic stacks

2. Corepresentability of RHom-functors

3. Finitely generated and coherent functors

4. Coherence of Hom-functors: flat case

5. Coherence of Hom-functors: non-flat case

6. Applications

References

\section{DeRIVED CATEGORIES OF SHEAVES ON ALGEBRAIC STACKS}

In this section, we review derived categories of sheaves on algebraic stacks. Fix an algebraic stack $X$. We take $\operatorname{Mod}(X)($ resp. $\mathbf{Q C o h}(X))$ to denote the abelian category of $\mathcal{O}_{X}$-modules (resp. quasicoherent $\mathcal{O}_{X}$-modules) on the lisse-étale site of $X[\mathrm{LMB}, 12.1]$. Take $\mathrm{D}(X)$ (resp. $\mathrm{D}_{\mathrm{QCoh}}(X)$ ) to denote the unbounded derived category of $\operatorname{Mod}(X)$ (resp. the full subcategory of $\mathrm{D}(X)$ with cohomology in $\mathrm{QCoh}(X))$. Superscripts such as,,$+- \geq n$, and $b$ decorating $\mathrm{D}(X)$ and $\mathrm{D}_{\mathrm{QCoh}}(X)$ should be interpreted as usual. In addition, if $X$ is locally noetherian, one may consider the category of coherent sheaves $\mathbf{C o h}(X)$ and the derived category $\mathrm{D}_{\mathrm{Coh}}(X)$.

If $X$ is a Deligne-Mumford stack, there is an associated small étale site. We take $\operatorname{Mod}\left(X_{\text {ét }}\right)\left(\operatorname{resp} . \mathbf{Q C o h}\left(X_{\text {ét }}\right)\right)$ to denote the abelian category of $\mathcal{O}_{X_{\text {ét }}}$-modules (resp. quasicoherent $\mathcal{O}_{X_{\text {ét }}}$-modules). There are naturally induced morphisms of abelian categories $\operatorname{Mod}(X) \rightarrow \operatorname{Mod}\left(X_{\text {ét }}\right)$ and $\mathbf{Q C o h}(X) \rightarrow \mathbf{Q C o h}\left(X_{\text {ét }}\right)$. Set $\mathrm{D}_{\mathrm{QCoh}}\left(X_{\text {ét }}\right)$ to be the triangulated category $\mathrm{D}_{\mathrm{QCoh}\left(X_{\text {ét }}\right)}\left(\operatorname{Mod}\left(X_{\text {ét }}\right)\right)$. Then, the natural functor $\mathrm{D}_{\mathrm{QCoh}}(X) \rightarrow \mathrm{D}_{\mathrm{QCoh}}\left(X_{\text {ét }}\right)$ is an equivalence of categories. If $X$ is a scheme, the corresponding statement for the Zariski site is also true.

For generalities on unbounded derived categories on ringed sites, we refer the reader to [KS06, §18.6]. We now record for future reference some useful formulae. If $\mathcal{M}^{\bullet}$ and $\mathcal{N}^{\bullet} \in \mathrm{D}(X)$, then there is the derived tensor product $\mathcal{M}^{\bullet} \otimes_{\mathcal{O}_{X}}^{\mathrm{L}} \mathcal{N}^{\bullet} \in \mathrm{D}(X)$, 
the derived sheaf Hom functor $\mathrm{RHom}_{\mathcal{O}_{X}}\left(\mathcal{M}^{\bullet}, \mathcal{N}^{\bullet}\right) \in \mathrm{D}(X)$ and the derived global Hom functor $\operatorname{RHom}_{\mathcal{O}_{X}}\left(\mathcal{M}^{\bullet}, \mathcal{N}^{\bullet}\right) \in \mathrm{D}(\mathbf{A b})$. For all $\mathcal{P}^{\bullet} \in \mathrm{D}(X)$ we have a functorial isomorphism:

$$
\operatorname{Hom}_{\mathcal{O}_{X}}\left(\mathcal{M} \bullet \otimes_{\mathcal{O}_{X}}^{\llcorner} \mathcal{N}^{\bullet}, \mathcal{P}^{\bullet}\right) \cong \operatorname{Hom}_{\mathcal{O}}\left(\mathcal{M}^{\bullet}, \operatorname{RHom}_{\mathcal{O}_{X}}\left(\mathcal{N}^{\bullet}, \mathcal{P}^{\bullet}\right)\right)
$$

as well as a functorial quasi-isomorphism:

$$
\mathrm{RH}^{-} \operatorname{Hom}_{\mathcal{O}_{X}}\left(\mathcal{M}^{\bullet} \otimes_{\mathcal{O}_{X}}^{\mathrm{L}} \mathcal{N}^{\bullet}, \mathcal{P}^{\bullet}\right) \simeq \mathrm{RHom}_{\mathcal{O}_{X}}\left(\mathcal{M}^{\bullet}, \operatorname{RHom}_{\mathcal{O}_{X}}\left(\mathcal{N}^{\bullet}, \mathcal{P}^{\bullet}\right)\right)
$$

Set $\mathrm{R} \Gamma(X,-)=\mathrm{RHom}_{\mathcal{O}_{X}}\left(\mathcal{O}_{X},-\right)$, then there is also a natural quasi-isomorphism:

$$
\operatorname{RHom}_{\mathcal{O}_{X}}\left(\mathcal{M}^{\bullet}, \mathcal{N}^{\bullet}\right) \simeq \operatorname{R\Gamma RHom} \mathcal{O}_{X}\left(\mathcal{M}^{\bullet}, \mathcal{N}^{\bullet}\right) \text {. }
$$

If $\mathcal{M}^{\bullet}, \mathcal{N}^{\bullet} \in \mathrm{D}_{\mathrm{QCoh}}(X)$, then $\mathcal{M}^{\bullet} \otimes_{\mathcal{O}_{X}}^{\llcorner} \mathcal{N}^{\bullet} \in \mathrm{D}_{\mathrm{QCoh}}(X)$. If $X$ is locally noetherian and $\mathcal{M}^{\bullet}, \mathcal{N}^{\bullet} \in \mathrm{D}_{\text {Coh }}^{-}(X)$, then $\mathcal{M}^{\bullet} \otimes_{\mathcal{O}_{X}}^{\mathrm{L}} \mathcal{N}^{\bullet} \in \mathrm{D}_{\text {Coh }}^{-}(X)$. Also, if $X$ is locally noetherian

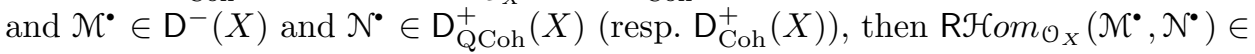
$\mathrm{D}_{\mathrm{QCoh}}^{+}(X)$ (resp. $\left.\mathrm{D}_{\mathrm{Coh}}^{+}(X)\right)$. These results are all consequences of [Ols07, §6] and LO08, §2].

Fix a morphism of algebraic stacks $f: X \rightarrow Y$. We let $\mathrm{R} f_{*}: \mathrm{D}(X) \rightarrow \mathrm{D}(Y)$ denote the derived functor of $f_{*}: \operatorname{Mod}(X) \rightarrow \operatorname{Mod}(Y)$. For $\mathcal{M}^{\bullet}, \mathcal{N}^{\bullet} \in \mathrm{D}(X)$ and $q \in \mathbb{Z}$, set:

$$
\mathcal{E} x t^{q}\left(f ; \mathcal{M}^{\bullet}, \mathcal{N}^{\bullet}\right):=\mathcal{H}^{q}\left(\mathrm{R} f_{*} \operatorname{RHom}_{\mathcal{O}_{X}}\left(\mathcal{M}^{\bullet}, \mathcal{N}^{\bullet}\right)\right) .
$$

If the morphism $f$ is quasicompact and quasiseparated, then the restriction of $\mathrm{R} f_{*}$ to $\mathrm{D}_{\mathrm{QCoh}}^{+}(X)$ induces a functor $\mathrm{R}_{*}: \mathrm{D}_{\mathrm{QCoh}}^{+}(X) \rightarrow \mathrm{D}_{\mathrm{QCoh}}^{+}(Y)$ Ols07, Lem. 6.20]. If $X$ and $Y$ are Deligne-Mumford stacks, then the restriction of $\mathrm{R}_{*}$ to $\mathrm{D}_{\mathrm{QCoh}}^{+}(X)$ coincides with the restriction of the derived functor of $\left(f_{\text {ét }}\right)_{*}: \operatorname{Mod}\left(X_{\text {ét }}\right) \rightarrow \operatorname{Mod}\left(Y_{\text {ét }}\right)$ to $\mathrm{D}_{\mathrm{QCoh}}\left(X_{\text {ét }}\right)$. A consequence of [LO08, Ex. 2.1.11] is that if in addition $f$ is representable, then the restriction of $\mathrm{R} f_{*}$ to $\mathrm{D}_{\mathrm{QCoh}}(X)$ induces a functor $\mathrm{R} f_{*}$ : $\mathrm{D}_{\mathrm{QCoh}}(X) \rightarrow \mathrm{D}_{\mathrm{QCoh}}(Y)$.

A morphism of algebraic stacks $f: X \rightarrow Y$, however, does not necessarily induce a left exact morphism of corresponding lisse-étale sites [Beh03, 5.3.12], thus the construction of the correct derived functors of $f^{*}: \mathbf{Q C o h}(Y) \rightarrow \mathbf{Q C o h}(X)$ is somewhat subtle. There are currently two approaches to constructing these functors. The first, due to M. Olsson [Ols07] and Y. Laszlo and M. Olsson [LO08, uses cohomological descent. The other approach appears in the Stacks Project Stacks. The latter approach is more widely applicable, but uses a completely different formulation (big sites) and requires significant amounts of technology that many people may not be familiar with.

In this article, where there is always some finiteness at hand, the approach of Olsson and Laszlo-Olsson is sufficient, thus is the method that we will employ. In any case, there exists a functor $\mathrm{L} f_{\mathrm{Q}}^{*}: \mathrm{D}_{\mathrm{QCoh}}(Y) \rightarrow \mathrm{D}_{\mathrm{QCoh}}(X)$ such that $\mathcal{H}^{0}\left(\mathrm{~L} f_{\mathrm{Q}}^{*} \mathcal{J}[0]\right) \cong f^{*} \mathcal{J}$, whenever $\mathcal{J} \in \mathbf{Q C o h}(Y)$. In addition, if $f$ is quasicompact and quasiseparated, $\mathfrak{J} \bullet \in \mathrm{D}_{\mathrm{QCoh}}(Y)$, and $\mathcal{N}^{\bullet} \in \mathrm{D}_{\mathrm{QCoh}}^{+}(X)$, then there is a natural isomorphism:

$$
\operatorname{Hom}_{\mathcal{O}_{Y}}\left(\mathcal{J}^{\bullet}, R f_{*} \mathcal{N}^{\bullet}\right) \cong \operatorname{Hom}_{\mathcal{O}_{X}}\left(\mathrm{~L} f_{\mathrm{Q}}^{*} \mathcal{J}^{\bullet}, \mathcal{N}^{\bullet}\right)
$$

Thus, $\mathrm{L} f_{\mathrm{Q}}^{*}$ is the left adjoint of $\mathrm{R} f_{*}$. In the situation where $X$ and $Y$ are DeligneMumford stacks, there also exists a derived functor $\mathrm{L} f_{\text {ét }}^{*}: \mathrm{D}_{\mathrm{QCoh}}\left(Y_{\text {ét }}\right) \rightarrow \mathrm{D}_{\mathrm{QCoh}}\left(X_{\text {ét }}\right)$. The restriction of $\mathrm{L} f_{\text {êt }}^{*}$ to $\mathrm{D}_{\mathrm{QCoh}}\left(Y_{\text {ét }}\right)$ coincides with $\mathrm{L} f_{\mathrm{Q}}^{*}$. 
If $f: X \rightarrow Y$ is a quasicompact morphism of locally noetherian algebraic stacks, $\mathrm{J}^{\bullet} \in \mathrm{D}_{\mathrm{Coh}}^{-}(Y)$, and $\mathcal{N}^{\bullet} \in \mathrm{D}_{\mathrm{QCoh}}^{+}(X)$, the isomorphism (1.4) is readily strengthened to a natural quasi-isomorphism in $\mathrm{D}_{\mathrm{QCoh}}^{+}(Y)$ :

$$
\mathrm{RH} \operatorname{Com}_{\mathcal{O}_{Y}}\left(\mathcal{J}^{\bullet}, \mathrm{R} f_{*} \mathcal{N}^{\bullet}\right) \simeq \mathrm{R} f_{*} \mathrm{R \mathcal {H }} \operatorname{Com}_{\mathcal{O}_{X}}\left(\mathrm{~L} f_{\mathrm{Q}}^{*} \mathcal{J}^{\bullet}, \mathcal{N}^{\bullet}\right) .
$$

If $f: X \rightarrow Y$ is a representable and quasicompact morphism of algebraic stacks, then the isomorphism (1.4) can be extended to all $\mathcal{N}^{*} \in \mathrm{D}_{\mathrm{QCoh}}(X)$. In this situation - which is covered in [HR12C, generalizing [Nee96, Prop. 5.3] - there is also the projection formula, which gives a functorial quasi-isomorphism for all $\mathcal{N}^{\bullet} \in \mathrm{D}_{\mathrm{QCoh}}(X)$ and $\mathcal{J}^{\bullet} \in \mathrm{D}_{\mathrm{QCoh}}(Y)$ :

$$
\left(\mathrm{R} f_{*} \mathcal{N}^{\bullet}\right) \otimes_{\mathcal{O}_{Y}}^{\mathrm{L}} \mathrm{J}^{\bullet} \simeq \mathrm{R} f_{*}\left(\mathcal{N}^{\bullet} \otimes_{\mathcal{O}_{X}}^{\mathrm{L}} \mathrm{L} f_{\mathrm{Q}}^{*} \mathrm{~J}^{\bullet}\right)
$$

For a morphism of algebraic stacks $f: X \rightarrow Y$ a complex $\mathcal{N}^{\bullet} \in \mathrm{D}_{\mathrm{QCoh}}^{-}(X)$ has finite tor-dimension over $Y$ if there exists a non-negative integer $n$ such that for all $i \in \mathbb{Z}$ and $\mathcal{J}^{\bullet} \in \mathrm{D}_{\mathrm{Q} C o h}^{\geq i}(Y)$ we have that $\mathcal{N}^{\bullet} \otimes_{\mathcal{O}_{X}}^{\mathrm{L}} \mathrm{L} f_{\mathrm{Q}}^{*} \mathfrak{J}^{\bullet} \in \mathrm{D}_{\mathrm{QCoh}}^{\geq i-n}(X)$. We conclude this section with the following easily proven lemma.

Lemma 1.1. Fix an affine noetherian scheme $S$ and a morphism of noetherian algebraic stacks $f: X \rightarrow S$. Let $\mathcal{M}^{\bullet} \in \mathrm{D}_{\text {Coh }}^{-}(X)$ and $\mathcal{N}^{\bullet} \in \mathrm{D}_{\text {Coh }}^{b}(X)$. If $\mathcal{N}^{\bullet}$ has finite tor-dimension over $S$, then the following functor preserves filtered colimits:

$$
\operatorname{Hom}_{\mathcal{O}_{X}}\left(\mathcal{M}^{\bullet}, \mathcal{N}^{\bullet} \otimes_{\mathcal{O}_{X}}^{\mathrm{L}} \mathrm{L} f_{\mathrm{Q}}^{*}(-)\right): \mathbf{Q} \mathbf{C o h}(S) \rightarrow \mathbf{A b} .
$$

We now briefly review homotopy limits in a triangulated category $\mathcal{T}$ admitting countable products. Fix for each $i \geq 0$ a morphism in $\mathcal{T}, t_{i}: T_{i+1} \rightarrow T_{i}$. Set $t: \Pi_{i>0} T_{i} \rightarrow \Pi_{i>0} T_{i}$ to be the composition of the product of the morphisms $t_{i}$ with the projection $\Pi_{i \geq 0} T_{i} \rightarrow \Pi_{i \geq 1} T_{i}$. We define holim $T_{i}$ via the following distinguished triangle:

$$
\underset{i}{\operatorname{holim}} T_{i} \longrightarrow \prod_{i \geq 0} T_{i} \stackrel{\mathrm{Id}-t}{\longrightarrow} \prod_{i \geq 0} T_{i} .
$$

The category of lisse-étale $\mathcal{O}_{X}$-modules is a Grothendieck abelian category, thus $\mathrm{D}(X)$ admits small products. Moreover, we wish to point out that the functors $\mathrm{R} \Gamma(X,-), \mathrm{RH} \operatorname{com}_{\mathcal{O}_{X}}\left(\mathcal{M}^{\bullet},-\right)$ for $\mathcal{M}^{\bullet} \in \mathrm{D}(X)$, and $\mathrm{R} f_{*}$ for a morphism of algebraic stacks $f: X \rightarrow Y$ all preserve homotopy limits because they preserve products.

The following result is well-known and appears in [Stacks, 08IY] (albeit in a slightly different, but equivalent formulation).

Lemma 1.2. Let $X$ be an algebraic stack and fix $\mathcal{N}^{\bullet} \in \mathrm{D}(X)$. Then, the projections $\mathcal{N}^{\bullet} \rightarrow \tau^{\geq-i} \mathcal{N}^{\bullet}$ induce a non-canonical morphism:

$$
\phi: \mathcal{N}^{\bullet} \rightarrow \underset{i}{\operatorname{holim}} \tau^{\geq-i} \mathcal{N}^{\bullet}
$$

If $\mathcal{N}^{\bullet} \in \mathrm{D}_{\mathrm{QCoh}}(X)$, then any such $\phi$ is a quasi-isomorphism.

Note that the main result of [Nee11] produces - in positive characteristic - complexes $\mathcal{N}^{\bullet} \in \mathrm{D}\left(\mathbf{Q C o h}\left(B \mathbb{N}_{a}\right)\right)$ with the property that there are no quasi-isomorphisms:

$$
\mathcal{N}^{\bullet} \rightarrow \underset{i}{\operatorname{holim}} \tau^{\geq-i} \mathcal{N}^{\bullet} .
$$

We wish to emphasize that this does not contradict Lemma 1.2. Indeed, while the categories $\mathrm{D}^{+}\left(\mathbf{Q} \operatorname{Coh}\left(B \mathbb{G}_{a}\right)\right)$ and $\mathrm{D}_{\mathrm{QCoh}}^{+}\left(B \mathbb{G}_{a}\right)$ are equivalent [Lur04, Thm. 3.8], this equivalence does not extend to the unbounded derived categories. 


\section{Corepresentability of RHom-Functors}

In this section we prove Theorem $\mathrm{B}$. Before we get to this we require the following two easily proven lemmas. Our first lemma gives two important maps used to prove Theorem B.

Lemma 2.1. Fix a morphism of noetherian algebraic stacks $f: X \rightarrow S$. Let $\mathcal{N}^{\bullet} \in$ $\mathrm{D}_{\mathrm{Coh}}^{-}(X)$ have finite tor-dimension over $S, \mathcal{F}^{\bullet} \in \mathrm{D}_{\mathrm{Coh}}^{-}(S)$, and $\mathcal{G}^{\bullet} \in \mathrm{D}_{\mathrm{QCoh}}^{+}(S)$.

(1) There is a natural morphism in $\mathrm{D}_{\mathrm{QCoh}}^{+}(S)$ :

$$
\mathrm{RH}_{\mathcal{H}} m_{\mathcal{O}_{S}}\left(\mathcal{F}^{\bullet}, \mathcal{G}^{\bullet}\right) \rightarrow \mathrm{R}_{*} \mathrm{RH} \operatorname{Com}_{\mathcal{O}_{X}}\left(\mathcal{N}^{\bullet} \otimes_{\mathcal{O}_{X}}^{\mathrm{L}} \mathrm{L} f_{\mathrm{Q}}^{*} \mathcal{F}^{\bullet}, \mathcal{N}^{\bullet} \otimes_{\mathcal{O}_{X}}^{\mathrm{L}} \mathrm{L} f_{\mathrm{Q}}^{*} \mathcal{G}^{\bullet}\right)
$$

(2) There is a natural quasi-isomorphism in $\mathrm{D}_{\mathrm{QCoh}}^{+}(X)$ :

$$
\mathcal{N}^{\bullet} \otimes_{\mathcal{O}_{X}}^{\mathrm{L}} \mathrm{L} f_{\mathrm{Q}}^{*} \mathrm{R} \mathcal{H} \operatorname{Hom}_{\mathcal{O}_{S}}\left(\mathcal{F}^{\bullet}, \mathcal{G}^{\bullet}\right) \simeq \mathrm{R} \mathcal{H} \operatorname{fom}_{\mathcal{O}_{X}}\left(\mathrm{~L} f_{\mathrm{Q}}^{*} \mathcal{F}^{\bullet}, \mathcal{N}^{\bullet} \otimes_{\mathcal{O}_{X}}^{\mathrm{L}} \mathrm{L} f_{\mathrm{Q}}^{*} \mathcal{G}^{\bullet}\right)
$$

Our next lemma will give the compatibility of the corepresenting object in Theorem [B] with base change - the result for schemes boils down to the well-known tor-independent base change $\left[\mathrm{FGI}^{+} 05\right.$, Thm. 8.3.2].

Lemma 2.2. Fix a 2-cartesian square of noetherian algebraic stacks:

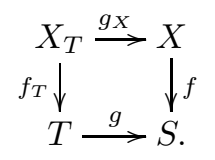

Let $\mathcal{M}^{\bullet} \in \mathrm{D}_{\text {Coh }}^{-}(X), \mathcal{N} \in \operatorname{Coh}(X)$, and $\mathcal{J}^{\bullet} \in \mathrm{D}_{\mathrm{QCoh}}^{+}(T)$. If $\mathcal{N}$ is flat over $S$, then there is a natural quasi-isomorphism in $\mathrm{D}_{\mathrm{QCoh}}^{+}(S)$ :

$$
\begin{aligned}
& \mathrm{R} f_{*} \mathrm{R} \mathcal{H} \operatorname{Com}_{\mathcal{O}_{X}}\left(\mathcal{M} \cdot, \mathcal{N}[0] \otimes_{\mathcal{O}_{X}}^{\mathrm{L}} \mathrm{L} f_{\mathrm{Q}}^{*} \mathrm{R} g_{*} \mathcal{J}^{\bullet}\right) \\
& \rightarrow \mathrm{R} g_{*} \mathrm{R}\left(f_{T}\right)_{*} \mathrm{R} \mathcal{H} \operatorname{Hom}_{\mathcal{O}_{X_{T}}}\left(\mathrm{~L}\left(g_{X}\right)_{\mathrm{Q}}^{*} \mathcal{M} \bullet,\left(g_{X}^{*} \mathcal{N}\right)[0] \otimes_{\mathcal{O}_{X_{T}}}^{\mathrm{L}} \mathrm{L}\left(f_{T}\right)_{\mathrm{Q}}^{*} \mathcal{J}^{\bullet}\right) \text {. }
\end{aligned}
$$

We can now prove Theorem B.

Proof of Theorem $B$. For background material on dualizing complexes we refer the reader to Har66, V.2]. For the convenience of the reader, however, we will recall the relevant results. A complex $\mathcal{K}^{\bullet} \in \mathrm{D}_{\text {Coh }}^{b}(S)$ is dualizing if it is locally of finite injective dimension and for any $\mathcal{F}^{\bullet} \in \mathrm{D}_{\mathrm{Coh}}(S)$, the natural map:

$$
\mathcal{F}^{\bullet} \rightarrow \mathrm{RHom}_{\mathcal{O}_{S}}\left(\mathrm{R} \mathcal{H} \operatorname{Hom}_{\mathcal{O}_{S}}\left(\mathcal{F}^{\bullet}, \mathcal{K}^{\bullet}\right), \mathcal{K}^{\bullet}\right)
$$

is a quasi-isomorphism. For notational convenience we set $\mathfrak{D}(-)=\mathrm{R} \mathcal{H} \operatorname{Com}_{\mathcal{O}_{S}}\left(-, \mathcal{K}^{\bullet}\right)$. Two useful facts about dualizing complexes are the following:

(1) the functor $\mathfrak{D}(-)$ interchanges $\mathrm{D}_{\text {Coh }}^{-}(S)$ and $\mathrm{D}_{\text {Coh }}^{+}(S)$;

(2) for $\mathcal{F}^{\bullet}, \mathcal{G}^{\bullet} \in \mathrm{D}_{\mathrm{Coh}}(S)$, there is a natural quasi-isomorphism:

$$
\mathrm{RH} \operatorname{lom}_{\mathcal{O}_{S}}\left(\mathcal{F}^{\bullet}, \mathcal{G}^{\bullet}\right) \simeq \mathrm{RH}^{\circ} m_{\mathcal{O}_{S}}\left(\mathfrak{D}\left(\mathcal{G}^{\bullet}\right), \mathfrak{D}\left(\mathcal{F}^{\bullet}\right)\right)
$$


Now fix $\mathrm{J}^{\bullet} \in \mathrm{D}_{\mathrm{Coh}}^{+}(S)$, then we have the following sequence of natural quasiisomorphisms:

$$
\begin{aligned}
& \mathrm{R} f_{*} \mathrm{RHom}_{\mathcal{O}_{X}}\left(\mathcal{M} \boldsymbol{M}^{\bullet}, \mathcal{N}^{\bullet} \otimes_{\mathcal{O}_{X}}^{\mathrm{L}} \mathrm{L} f_{\mathrm{Q}}^{*} \mathcal{J}^{\bullet}\right) \\
& \simeq R f_{*} \operatorname{RHom}_{\mathcal{O}_{X}}\left(\mathcal{M} \cdot \mathfrak{N}^{\bullet}, \mathcal{N}^{\bullet} \otimes_{\mathcal{O}_{X}}^{\mathrm{L}} \mathrm{L} f_{\mathrm{Q}}^{*} \mathrm{RH} \mathcal{H}_{\mathcal{O}_{S}}\left(\mathfrak{D}\left(\mathcal{J}^{\bullet}\right), \mathcal{K}^{\bullet}\right)\right)
\end{aligned}
$$

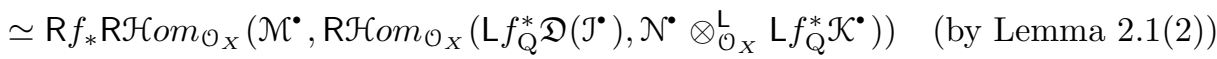

$$
\begin{aligned}
& \simeq R f_{*} \mathrm{RH} \mathcal{H}_{0} m_{\mathcal{O}_{X}}\left(\mathcal{M} \cdot \otimes_{\mathcal{O}_{X}}^{\mathrm{L}} \mathrm{L} f_{\mathrm{Q}}^{*} \mathfrak{D}\left(\mathcal{J}^{\bullet}\right), \mathcal{N}^{\bullet} \otimes_{\mathcal{O}_{X}}^{\mathrm{L}} \mathrm{L} f_{\mathrm{Q}}^{*} \mathcal{K}^{\bullet}\right) \quad \text { (by (1.2) } \\
& \left.\simeq \mathrm{R} f_{*} \mathrm{RH} \mathcal{H o m}_{\mathcal{O}_{X}}\left(\mathrm{~L} f_{\mathrm{Q}}^{*} \mathfrak{D}\left(\mathcal{J}^{\bullet}\right), \mathrm{RH} \mathcal{H o m}_{\mathcal{O}_{X}}\left(\mathcal{M} \cdot \mathcal{N}^{\bullet} \otimes_{\mathcal{O}_{X}}^{\mathrm{L}} \mathrm{L} f_{\mathrm{Q}}^{*} \mathcal{K}^{\bullet}\right)\right) \quad \text { (by (1.2) }\right)
\end{aligned}
$$

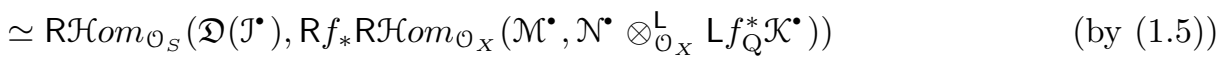

$$
\begin{aligned}
& \left.\simeq \mathrm{RH}_{\mathcal{H}} m_{\mathcal{O}_{S}}\left(\mathfrak{D}\left(\mathrm{R}_{*} \mathrm{RH} \mathcal{H o m}_{\mathcal{O}_{X}}\left(\mathcal{M}^{\bullet}, \mathcal{N}^{\bullet} \otimes_{\mathcal{O}_{X}}^{\mathrm{L}} \mathrm{L} f_{\mathrm{Q}}^{*} \mathcal{K}^{\bullet}\right)\right), \mathcal{J}^{\bullet}\right) \quad \text { (by (2) }\right) \text {. }
\end{aligned}
$$

The final quasi-isomorphism is a consequence of the following sequence of observations. First, $\mathcal{N}^{\bullet} \in \mathrm{D}_{\text {Coh }}^{b}(X)$ has finite tor-dimension over $S$ and $\mathcal{K}^{\bullet} \in \mathrm{D}_{\text {Coh }}^{b}(S)$, so $\mathcal{N}^{\bullet} \otimes_{\mathcal{O}_{X}}^{\llcorner} \mathrm{L} f_{\mathrm{Q}}^{*} \mathcal{K}^{\bullet} \in \mathrm{D}_{\text {Coh }}^{b}(X)$. Also, $f$ is proper so [Ols07, 6.4.4 \& 10.13] implies that $\mathrm{R} f_{*} \mathrm{RH}_{\mathcal{H}} \operatorname{lom}_{\mathcal{O}_{X}}\left(\mathcal{M}^{\bullet}, \mathcal{N}^{\bullet} \otimes_{\mathcal{O}_{X}}^{\mathrm{L}} \mathrm{L} f_{\mathrm{Q}^{*}}^{*} \mathcal{K}^{\bullet}\right) \in \mathrm{D}_{\text {Coh }}^{+}(S)$. Thus (2) applies. Hence, we have produced a natural quasi-isomorphism for all $\mathfrak{J}^{\bullet} \in \mathrm{D}_{\text {Coh }}^{+}(S)$ :

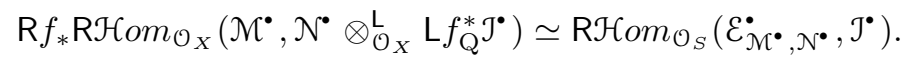

where

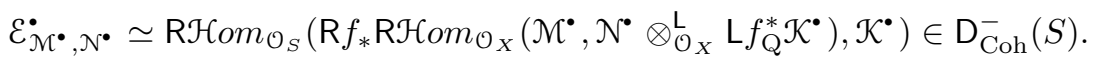

We now need to extend the quasi-isomorphism (2.1) to $\mathcal{J}^{\bullet} \in \mathrm{D}_{\mathrm{QCoh}}^{+}(S)$. First, we note that because $\mathcal{N}^{\bullet}$ is bounded, there exists an $r$ such that for all $n$ and all $\mathrm{J} \cdot \in \mathrm{D}_{\mathrm{QCoh}}(S)$ the natural map:

$$
\tau^{\geq n+r}\left(\mathcal{N}^{\bullet} \otimes_{\mathcal{O}_{X}}^{\mathrm{L}} \mathrm{L} f_{\mathrm{Q}}^{*} \mathrm{~J}^{\bullet}\right) \rightarrow \tau^{\geq n+r}\left(\mathcal{N}^{\bullet} \otimes_{\mathcal{O}_{X}}^{\mathrm{L}} \mathrm{L} f_{\mathrm{Q}}^{*}\left[\tau^{\geq n} \mathcal{J}^{\bullet}\right]\right)
$$

is a quasi-isomorphism. Hence, by Lemma 1.2 there exist maps for all $\tau^{\bullet} \in \mathrm{D}_{\mathrm{Coh}}(S)$ :

$$
\begin{aligned}
& \operatorname{RHom}_{\mathcal{O}_{S}}\left(\mathcal{E}_{\mathcal{N}^{\bullet}, \mathfrak{N}^{\bullet}, \mathcal{J}^{\bullet}}\right) \simeq \operatorname{holim}_{n} \operatorname{RHom} \mathcal{O}_{S}\left(\mathcal{E}_{\mathcal{M}^{\bullet}, \mathcal{N}^{\bullet}}, \tau^{\geq-n} \mathcal{J}^{\bullet}\right) \\
& \simeq \operatorname{holim}_{n} \mathrm{R} f_{*} \mathrm{RH} \mathcal{H o m}_{\mathcal{O}_{X}}\left(\mathcal{M H}^{*}, \mathcal{N}^{\bullet} \otimes_{\mathcal{O}_{X}}^{\mathrm{L}} \mathrm{L} f_{\mathrm{Q}}^{*}\left[\tau^{\geq-n} \mathcal{J}^{\bullet}\right]\right) \\
& \rightarrow \operatorname{holim}_{n} \operatorname{Rf}_{*} \operatorname{RH} \operatorname{fom}_{\mathcal{O}_{X}}\left(\mathcal{M} \cdot \tau^{\geq-n+r}\left(\mathcal{N}^{\bullet} \otimes_{\mathcal{O}_{X}}^{\mathrm{L}} \mathrm{L} f_{\mathrm{Q}}^{*}\left[\tau^{\geq-n} \mathcal{J}^{\bullet}\right]\right)\right) \\
& \simeq \operatorname{holim}_{n} \mathrm{R} f_{*} \mathrm{RH}_{\mathcal{H}} \operatorname{mom}_{\mathcal{O}_{X}}\left(\mathcal{M}^{\bullet}, \tau^{\geq-n+r}\left(\mathcal{N}^{\bullet} \otimes_{\mathcal{O}_{X}}^{\mathrm{L}} \mathrm{L} f_{\mathrm{Q}}^{*} \mathcal{J}^{\bullet}\right)\right) \\
& \simeq \mathrm{R}_{*} \mathrm{RH} \mathcal{H o m}_{\mathcal{O}_{X}}\left(\mathcal{M} \bullet, \mathcal{N}^{\bullet} \otimes_{\mathcal{O}_{X}}^{\mathrm{L}} \mathrm{L} f_{\mathrm{Q}^{*}}^{*} \mathcal{J}^{\bullet}\right) \text {. }
\end{aligned}
$$

Note, however, that the maps above depend on $\mathcal{N}^{\bullet}, \mathcal{N}^{\bullet}$, and $\mathcal{J}^{\bullet}$ in a non-natural way (this is because holim is constructed as a cone, thus is not functorial). In any case, corresponding to the identity map $\varepsilon_{\mathcal{N} \cdot, \mathcal{N}^{\bullet}} \rightarrow \varepsilon_{\mathcal{M}^{\bullet}, \mathcal{N}^{\bullet}}$ there is a morphism $\psi_{\mathcal{M}^{\bullet}, \mathcal{N}^{\bullet}}: \mathcal{M}^{\bullet} \rightarrow \mathcal{N}^{\bullet} \otimes_{\mathcal{O}_{X}}^{\mathrm{L}} \mathrm{L} f_{\mathrm{Q}^{*}}^{*} \mathcal{E}_{\mathcal{M}^{\bullet}, \mathcal{N}^{\bullet}}$ (which is not necessarily functorial in $\mathcal{M}^{\bullet}$ or $\left.\mathcal{N}^{\bullet}\right)$. Now take $J^{\bullet} \in \mathrm{D}_{\mathrm{QCoh}}^{+}(S)$, then Lemma 2.1(1) provides a natural sequence of maps:

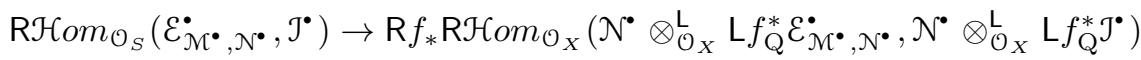

$$
\begin{aligned}
& \rightarrow \mathrm{R} f_{*} \mathrm{RH} \mathcal{H o m}_{\mathcal{O}_{X}}\left(\mathcal{M}^{\bullet}, \mathcal{N}^{\bullet} \otimes_{\mathcal{O}_{X}}^{\mathrm{L}} \mathrm{L} f_{\mathrm{Q}}^{*} \mathcal{J}^{\bullet}\right) \text {. }
\end{aligned}
$$

By (2.1), the map above is certainly a quasi-isomorphism for all $\mathrm{J}^{\bullet} \in \mathrm{D}_{\mathrm{Coh}}^{+}(S)$. To show that it is a quasi-isomorphism for all $\mathcal{J}^{\bullet} \in \mathrm{D}_{\mathrm{QCoh}}^{+}(S)$, by the "way-out right" 
results of [Har66, I.7.1], it is sufficient to prove that it is a quasi-isomorphism for all quasicoherent $\mathcal{O}_{S}$-modules. We may now reduce to the case where $S$ is an affine and noetherian scheme. Hence, it is sufficient to prove that the natural transformation of functors from $\mathbf{Q C o h}(S) \rightarrow \mathbf{A b}$ :

$$
\operatorname{Hom}_{\mathcal{O}_{S}}\left(\mathcal{E}_{\mathcal{N}^{\bullet}, \mathcal{N}^{\bullet},},(-)[0]\right) \rightarrow \operatorname{Hom}_{\mathcal{O}_{X}}\left(\mathcal{M} \bullet, \mathcal{N} \bullet \otimes_{\mathcal{O}_{X}}^{\mathrm{L}} \mathrm{L} f_{\mathrm{Q}}^{*}(-)[0]\right)
$$

is an isomorphism. By Lemma 1.1, both functors preserve filtered colimits and the exhibited natural transformation is an isomorphism for all $\mathcal{J} \in \mathbf{C o h}(S)$. Since any $\mathcal{J} \in \mathbf{Q C o h}(S)$ is a filtered colimit of objects of $\mathbf{C o h}(S)$, we deduce the result.

It now remains to address the compatibility of $\varepsilon_{\mathcal{M}}^{\bullet}, \mathcal{N}^{\bullet}$ with base change in the situation where $\mathcal{N}^{\bullet} \simeq \mathcal{N}[0]$ and $\mathcal{N} \in \operatorname{Coh}(X)$ is flat over $S$. So, we fix a morphism of noetherian algebraic stacks $g: T \rightarrow S$ such that $T$ admits a dualizing complex and form the 2-cartesian square of noetherian algebraic stacks:

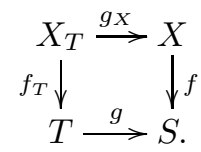

By Lemma 2.2 and what we have proven so far, there is a quasi-isomorphism, natural in $\mathcal{J}^{\bullet} \in \mathrm{D}_{\mathrm{QCoh}}^{+}(T)$ :

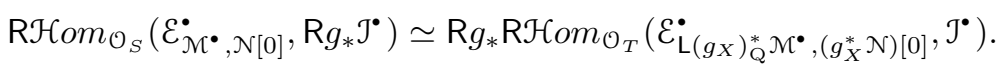

By trivial duality (1.5) we thus obtain a quasi-isomorphism, natural in $\mathcal{J}^{\bullet} \in \mathrm{D}_{\mathrm{QCoh}}^{+}(T)$ :

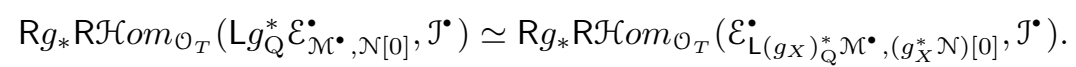

By (1.3), we thus see that we have a quasi-isomorphism, natural in $\mathcal{J} \bullet \mathrm{D}_{\mathrm{QCoh}}^{+}(T)$ :

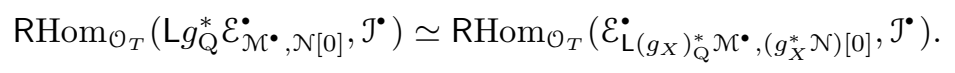

By Lemma 1.2 and the above we obtain a sequence of quasi-isomorphisms:

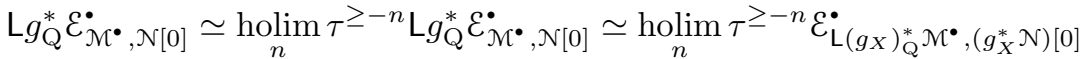

$$
\begin{aligned}
& \simeq \mathcal{E}_{\mathrm{L}\left(g_{X}\right)_{\mathrm{Q}}^{*} \mathcal{N} \mathfrak{M},\left(g_{X}^{*} \mathcal{N}\right)[0]} .
\end{aligned}
$$

\section{Finitely generated And COHEREnt FunCtors}

Fix a ring $A$. Let $\operatorname{Fun}_{A}^{\times}$denote the category of functors $\operatorname{Mod}(A) \rightarrow$ Sets which commute with finite products. This is a full subcategory of the category of all functors $\operatorname{Mod}(A) \rightarrow$ Sets. Denote by $\operatorname{Lin}_{A}$ the category of $A$-linear functors $\operatorname{Mod}(A) \rightarrow \operatorname{Mod}(A)$. The following is straightforward.

Lemma 3.1. Fix a ring $A$, then the forgetful functor:

$$
\operatorname{Lin}_{A} \rightarrow \operatorname{Fun}_{A}^{\times}
$$

is an equivalence of categories.

In particular, for a ring $A$, set $T=\operatorname{Spec} A$ and note that Lemma 3.1 shows that the category of additive functors $\mathbf{Q} \mathbf{C o h}(T) \rightarrow \mathbf{A b}$ is equivalent to the category of $A$-linear functors $\operatorname{Mod}(A) \rightarrow \operatorname{Mod}(A)$. We will use this equivalence without further mention to translate definitions between the two categories.

A functor $Q: \operatorname{Mod}(A) \rightarrow$ Sets is finitely generated if there exists an $A$-module $I$ and an object $\eta \in Q(I)$ such that for all $A$-modules $M$, the induced morphism 
of sets $\operatorname{Hom}_{A}(I, M) \rightarrow Q(M): f \mapsto f_{*} \eta$ is surjective. We call the pair $(I, \eta)$ a generator for the functor $Q$. The notion of finite generation of a functor is due to M. Auslander Aus66.

Example 3.2. Fix a ring $A$. For any $A$-module $I$, the functor $M \mapsto \operatorname{Hom}_{A}(I, M)$ is finitely generated. The functor $M \mapsto I \otimes_{A} M$ is finitely generated if and only if the $A$-module $I$ is finitely generated as an $A$-module. A generator is obtained by choosing a surjection $A^{n} \rightarrow I$, and noting that for any $A$-module $M$, there is a surjection $A^{n} \otimes_{A} M \rightarrow I \otimes_{A} M$, and an isomorphism $A^{n} \otimes_{A} M \rightarrow \operatorname{Hom}_{A}\left(A^{n}, M\right)$.

Example 3.3. Fix a ring $A$ and a collection of finitely generated functors $\left\{F_{\lambda}\right.$ : $\operatorname{Mod}(A) \rightarrow \mathbf{A b}\}_{\lambda \in \Lambda}$ indexed by a set $\Lambda$. Then, the functor $M \mapsto \Pi_{\lambda \in \Lambda} F_{\lambda}(M)$ is finitely generated. Indeed, for each $\lambda \in \Lambda$, let $\left(I_{\lambda}, \eta_{\lambda}\right)$ be a generator for $F_{\lambda}$. Then, $\left(\Pi_{\lambda \in \Lambda} I_{\lambda},\left(\eta_{\lambda}\right)_{\lambda \in \Lambda}\right)$ is a generator for the functor $M \mapsto \Pi_{\lambda \in \Lambda} F_{\lambda}(M)$.

For an $A$-algebra $B$, and a functor $Q: \operatorname{Mod}(A) \rightarrow$ Sets, there is an induced functor $Q_{B}: \operatorname{Mod}(B) \rightarrow$ Sets given by regarding a $B$-module as an $A$-module. Note that since the forgetful functor $\operatorname{Mod}(B) \rightarrow \operatorname{Mod}(A)$ commutes with all limits and colimits, it follows that if the functor $Q$ preserves certain limits or colimits, so does the functor $Q_{B}$. In particular, from Lemma 3.1 we see that if the functor $Q$ commutes with finite products, then the functor $Q_{B}: \operatorname{Mod}(B) \rightarrow$ Sets is canonically $B$-linear and thus defines a functor $Q_{B}: \operatorname{Mod}(B) \rightarrow \operatorname{Mod}(B)$. We will use this fact frequently and without further comment.

Example 3.4. For an $A$-algebra $B$, if $(I, \eta)$ generates $Q$, let $p: I \rightarrow I \otimes_{A} B$ be the natural $A$-module homomorphism, then $\left(I \otimes_{A} B, p_{*} \eta\right)$ generates $Q_{B}$.

An additive functor $F: \operatorname{Mod}(A) \rightarrow \mathbf{A b}$ is coherent, if there exists an $A$-module homomorphism $f: I \rightarrow J$ and an element $\eta \in F(I)$, inducing an exact sequence for any $A$-module $M$ :

$$
\operatorname{Hom}_{A}(J, M) \longrightarrow \operatorname{Hom}_{A}(I, M) \longrightarrow F(M) \longrightarrow 0 .
$$

We refer to the data $(f: I \rightarrow J, \eta)$ as a presentation for $F$. For accounts of coherent functors, we refer the interested reader to Aus66, Har98. We now have a number of examples.

Example 3.5. Given an exact sequence of additive functors $H_{i}: \operatorname{Mod}(A) \rightarrow \mathbf{A b}$ :

$$
\mathrm{H}_{1} \longrightarrow \mathrm{H}_{2} \longrightarrow \mathrm{H}_{3} \longrightarrow \mathrm{H}_{4} \longrightarrow \mathrm{H}_{5},
$$

where for $i \neq 3$, we have that $H_{i}$ is coherent. Then, $H_{3}$ is coherent. In particular, the category of coherent functors is stable under kernels, cokernels, subquotients, and extensions. This follows from [Aus66, Prop. 2.1].

Example 3.6. Fix a ring $A$ and an $A$-algebra $B$. If $F: \operatorname{Mod}(A) \rightarrow \mathbf{A b}$ is a coherent functor, then analogously to Example 3.4, the restriction $F_{B}: \operatorname{Mod}(B) \rightarrow$ $\mathrm{Ab}$ is also coherent.

Fix a ring $A$. A functor $F: \operatorname{Mod}(A) \rightarrow \operatorname{Mod}(A)$ is half-exact if for any short exact sequence of $A$-modules $0 \rightarrow M^{\prime} \rightarrow M \rightarrow M^{\prime \prime} \rightarrow 0$, the sequence $F\left(M^{\prime}\right) \rightarrow F(M) \rightarrow F\left(M^{\prime \prime}\right)$ is exact.

Example 3.7. Fix a ring $A$ and let $Q^{\bullet}$ be a complex of $A$-modules. Then the functor $M \mapsto H^{i}\left(\operatorname{Hom}_{A}\left(Q^{\bullet}, M\right)\right)$ is coherent for all $i \in \mathbb{Z}$. If the complex $Q^{\bullet}$ is term-by-term projective, the functor $M \mapsto H^{i}\left(\operatorname{Hom}_{A}\left(Q^{\bullet}, M\right)\right)$ is also half-exact. 
An $A$-linear functor of the form $M \mapsto \operatorname{Ext}_{A}^{i}\left(Q^{\bullet}, M\right)$ is said to be corepresentable by a complex. By Example 3.7, such functors are coherent and half-exact, and were intially studied by M. Auslander Aus66, with stronger results - in the noetherian setting - obtained R. Hartshorne Har98. In HR12a, it is shown that étale locally any half-exact, coherent functor is corepresentable by a complex.

Example 3.8. Fix a ring $A$ and a coherent functor $F: \operatorname{Mod}(A) \rightarrow \mathbf{A b}$. Then, $F$ preserves small products. It was shown by H. Krause [Kra03, Prop. 3.2] that the preservation of small products characterizes coherent functors.

Example 3.9. Fix a ring $A$. Example 3.3 extends to show that the category of coherent functors $\operatorname{Mod}(A) \rightarrow \mathbf{A b}$ is closed under small products. By Example 3.5. the category of coherent functors $\operatorname{Mod}(A) \rightarrow \mathbf{A b}$ is also closed under equalizers. Thus, the category of coherent functors $\operatorname{Mod}(A) \rightarrow \mathbf{A b}$ is closed under small limits.

Example 3.10. Fix a ring $A$ and a coherent functor $F: \operatorname{Mod}(A) \rightarrow \mathbf{A b}$ which is left exact. By Example 3.8, $F$ also preserves small products, thus $F$ preserves small limits. The Eilenberg-Watts Theorem Wat60, Thm. 6] now implies that $F$ is corepresentable. That is, there exists an $A$-module $Q$ such that $F(-) \cong$ $\operatorname{Hom}_{A}(Q,-)$. If, in addition, the functor $F$ preserves direct limits, then $Q$ is finitely presented. By Example 3.7 this observation generalizes [EGA, III.7.4.6].

Example 3.11. Fix a ring $A$ and an $A$-module $N$. Then the functor $M \mapsto M \otimes_{A} N$ is coherent if and only if the $A$-module $N$ is finitely presented.

Example 3.12. Fix a noetherian $\operatorname{ring} R$. Let $Q^{\bullet}$ be a complex of finitely generated $R$-modules, then the functor $M \mapsto H^{i}\left(Q^{\bullet} \otimes_{R} M\right)$ is coherent and commutes with filtered colimits. If the complex $Q^{\bullet}$ is, in addition, flat term-by-term, then the functor $M \mapsto H^{i}\left(Q^{\bullet} \otimes_{R} M\right)$ is also half-exact.

Example 3.13. Fix a noetherian $\operatorname{ring} R$. Let $Q^{\bullet}$ be a bounded above complex of $R$-modules with coherent cohomology. Then, the functors $M \mapsto \operatorname{Tor}_{i}^{R}\left(Q^{\bullet}, M\right)$ and $M \mapsto \operatorname{Ext}_{R}^{i}\left(Q^{\bullet}, M\right)$ are coherent, half-exact and preserve filtered colimits.

\section{Coherence of Hom-Functors: flat CASE}

Proof of Theorem $\mathrm{C}$ The proof of Theorem $\mathrm{C}$ will be via an induction argument that permits us to reduce to the case of Theorem $B$. The following notation will be useful.

Notation 4.1. For a morphism of algebraic stacks $f: X \rightarrow S$, and $\mathcal{M}^{\bullet}, \mathcal{N}^{\bullet} \in$ $\mathrm{D}_{\mathrm{QCoh}}(X)$, set:

$$
\mathbb{H}_{\mathcal{M} \cdot, \mathcal{N}^{\bullet}}:=\operatorname{Hom}_{\mathcal{O}_{X}}\left(\mathcal{M} \cdot \mathcal{N}^{\bullet} \otimes_{\mathcal{O}_{X}}^{\mathrm{L}} \mathrm{L} f_{\mathrm{Q}}^{*}(-)\right): \mathbf{Q C o h}(S) \rightarrow \mathbf{A b} .
$$

For $\mathcal{N}^{\bullet} \in \mathrm{D}_{\mathrm{QCoh}}(X)$ we set $\mathcal{T}_{X / S}^{\mathcal{N}^{\bullet}} \subset \mathrm{D}_{\mathrm{QCoh}}(X)$ to be the full subcategory having objects those $\mathcal{M}^{\bullet}$ with the property that $\mathbb{H}_{\mathcal{M}^{\bullet}, \mathcal{N}^{\bullet}[n]}$ is coherent for all $n \in \mathbb{Z}$.

We begin with two general reductions.

Lemma 4.2. Fix an affine scheme $S$ and a morphism of algebraic stacks $f: X \rightarrow S$ and $\mathcal{N}^{*} \in \mathrm{D}_{\mathrm{QCoh}}(X)$, then the subcategory $\mathcal{T}_{X / S}^{\mathcal{N}^{*}} \subset \mathrm{D}_{\mathrm{QCoh}}(X)$ is triangulated and closed under small direct sums. In particular, if

(1) $\mathrm{D}_{\mathrm{QCoh}}^{-}(X) \subset \mathcal{T}_{X / S}^{\mathcal{N}^{\bullet}}$, or

(2) $\mathcal{N}^{\bullet}$ has finite tor-dimension over $S$ and $\mathcal{M} \in \mathcal{T}_{X / S}^{\mathcal{N}^{\bullet}}$ for all $\mathcal{M} \in \mathbf{Q C o h}(X)$, 
then $\mathcal{T}_{X / S}^{\mathcal{N}^{\bullet}}=\mathrm{D}_{\mathrm{QCoh}}(X)$.

Proof. Certainly, $\mathcal{T}_{X / S}^{\mathcal{N}^{\bullet}}$ is closed under shifts. Next, given a triangle $\mathcal{M}_{1}^{\bullet} \rightarrow \mathcal{M}_{2}^{\bullet} \rightarrow$ $\mathcal{M}_{3}^{\bullet}$ in $\mathrm{D}_{\mathrm{QCoh}}(X)$ with $\mathcal{M}_{1}^{*}, \mathcal{M}_{2}^{*} \in \mathcal{T}_{X / S}^{\mathcal{N}^{*}}$, we obtain an exact sequence of functors:

$$
\mathbb{H}_{\mathcal{M}_{2}^{\bullet}[1], \mathcal{N}^{\bullet}} \rightarrow \mathbb{H}_{\mathcal{M}_{1}^{\bullet}[1], \mathcal{N}^{\bullet}} \rightarrow \mathbb{H}_{\mathcal{M}_{3}^{\bullet}, \mathcal{N}^{\bullet}} \rightarrow \mathbb{H}_{\mathcal{M}_{2}^{\bullet}, \mathfrak{N}^{\bullet}} \rightarrow \mathbb{H}_{\mathcal{M}_{1}^{\bullet}, \mathcal{N}^{\bullet}}
$$

By Example 3.5, $\mathbb{H}_{\mathcal{M}_{3}^{\bullet}, \mathcal{N}^{\bullet}} \in \mathcal{T}_{X / S}^{\mathcal{N}^{\bullet}}$ and so $\mathcal{T}_{X / S}^{\mathcal{N}^{\bullet}}$ is a triangulated subcategory of $\mathrm{D}_{\mathrm{QCoh}}(X)$. Let $\left\{\mathcal{M}_{i}^{\bullet}\right\}_{i \in I}$ be a set of elements from $\mathcal{T}_{X / S}^{\mathcal{N}^{\bullet}}$. Set $\mathcal{M}^{\bullet}=\oplus_{i \in I} \mathcal{M}_{i}^{\bullet}$, then for all $n \in \mathbb{Z}$ there is an isomorphism of functors $\mathbb{H}_{\mathcal{N}^{\bullet}, \mathbb{N}^{\bullet}[n]} \cong \Pi_{i \in I} \mathbb{H}_{\mathcal{N}_{i}, \mathfrak{N} \bullet[n]}$. By Example 3.9 we conclude that $\mathcal{M}^{\bullet} \in \mathcal{T}_{X / S}^{\mathcal{N}^{*}}$.

For (11), by [LO08, Lem. 4.3.2], given $\mathcal{M}^{\bullet} \in \mathrm{D}_{\mathrm{QCoh}}(X)$, there is a triangle:

$$
\bigoplus_{n \geq 0} \tau^{\leq n} \mathcal{M} \cdot \rightarrow \bigoplus_{n \geq 0} \tau^{\leq n} \mathcal{M} \cdot \rightarrow \mathcal{M} \cdot
$$

By hypothesis, $\tau^{\leq n} \mathcal{M} \bullet \in \mathcal{T}_{X / S}^{\mathcal{N}^{\bullet}}$ for all $n \geq 0$, and so $\mathcal{M}^{\bullet} \in \mathcal{T}_{X / S}^{\mathcal{N}^{\bullet}}$.

For (2) by (11), it is sufficient to prove that $\mathrm{D}_{\mathrm{QCoh}}^{-}(X) \subset \mathcal{T}_{X / S}^{\mathcal{N}^{\bullet}}$. Since $\mathcal{N}^{\bullet}$ has finite tor-dimension over $S$, there exists an integer $l$ such that the natural map $\mathcal{N}^{\bullet} \otimes_{\mathcal{O}_{X}}^{\mathrm{L}} \mathrm{L} f_{\mathrm{Q}^{*}}^{*} I \in \mathrm{D}_{\mathrm{Q} C o h}^{\geq l}(X)$ for all $I \in \mathbf{Q C o h}(S)$. Thus, if $\mathcal{M}^{\bullet} \in \mathrm{D}_{\mathrm{QCoh}}^{-}(X)$, then for any integer $n$ we have a natural isomorphism of functors: $\mathbb{H}_{\mathcal{M} \cdot{ }^{\bullet}, \mathcal{N}^{\bullet}[n]} \cong$ $\mathbb{H}_{\tau \geq l-n} \mathcal{M}^{\bullet}, \mathcal{N}^{\bullet}[n]$. Hence, it is sufficient to prove that $\mathrm{D}_{\mathrm{QCoh}}^{b}(X) \subset \mathcal{T}_{X / S}^{\mathcal{N}^{\bullet}}$. Working with truncations and cones gives the result.

Lemma 4.3. Fix an affine scheme $S$, a representable morphism of algebraic $S$ stacks $p: X^{\prime} \rightarrow X$ which is quasicompact, and $\mathcal{G}^{\prime \bullet} \in \mathrm{D}_{\mathrm{QCoh}}\left(X^{\prime}\right)$. Then, $\mathbb{H}_{\mathcal{M}}{ }^{\bullet}, R_{*} \mathcal{G}^{\prime} \cdot=$ $\mathbb{H}_{\mathrm{L} p_{\mathrm{Q}}^{*} \mathcal{M}} \cdot \mathcal{G}^{\prime}$. In particular, if $\mathcal{T}_{X^{\prime} / S}^{\mathcal{G}^{\bullet}}=\mathrm{D}_{\mathrm{QCoh}}\left(X^{\prime}\right)$, then $\mathcal{T}_{X / S}^{\mathrm{R} p_{*} \mathcal{G}^{\bullet}}=\mathrm{D}_{\mathrm{Q} \text { Coh }}(X)$.

Proof. Fix $\mathcal{M} \bullet \in \mathrm{D}_{\mathrm{QCoh}}(X)$, then:

$$
\begin{aligned}
& \mathbb{H}_{\mathcal{N} \cdot{ }^{\bullet}, \mathrm{R} p_{*} \mathcal{G}^{\prime}}=\operatorname{Hom}_{\mathcal{O}_{X}}\left(\mathcal{M}^{\bullet},\left(R p_{*} \mathcal{G}^{\prime \bullet}\right) \otimes_{\mathcal{O}_{X}}^{\mathrm{L}} \mathrm{L} f_{\mathrm{Q}}^{*}(-)\right) \\
& \cong \operatorname{Hom}_{\mathcal{O}_{X}}\left(\mathcal{M} \cdot{ }^{\bullet}, R p_{*}\left(\mathcal{G}^{\prime \bullet} \otimes_{\mathcal{O}_{X^{\prime}}}^{\mathrm{L}} \mathrm{L} p_{\mathrm{Q}}^{*} \mathrm{~L} f_{\mathrm{Q}}^{*}(-)\right)\right)
\end{aligned}
$$

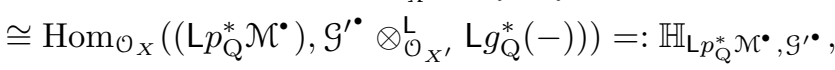

with the penultimate isomorphism given by the projection formula (1.6).

We now have our first induction result.

Lemma 4.4. Fix an affine scheme $S$, a morphism of algebraic stacks $f: X \rightarrow S$ which is of finite presentation, $\mathcal{N}^{\bullet} \in \mathrm{D}_{\mathrm{QCoh}}(X)$, and an integer $n \in \mathbb{Z}$. Suppose that the functor $\mathbb{H}_{\mathcal{M}, \mathfrak{N}^{\bullet}[r]}$ is coherent in the following situations:

(1) for all $r<n$ and $\mathcal{M} \in \mathbf{Q C o h}(X)$;

(2) $r=n$ and $\mathcal{M} \in \mathbf{Q C o h}(X)$ of finite presentation.

Then, the functor $\mathbb{H}_{\mathcal{M}, \mathfrak{N} \bullet[n]}$ is coherent for all $\mathcal{M} \in \mathbf{Q C o h}(X)$.

Proof. Fix $\mathcal{M} \in \mathbf{Q C o h}(X)$, then we must prove that $\mathbb{H}_{\mathcal{M}, \mathfrak{N} \bullet[n]}$ is coherent. The morphism $f$ is of finite presentation, so by $\operatorname{Ryd09}$, Thm. A], the quasicoherent $\mathcal{O}_{X}$-module $\mathcal{M}$ is a filtered colimit of $\mathcal{O}_{X}$-modules $\mathcal{M}_{\lambda}$ of finite presentation. Let $Q_{1}=\oplus_{\lambda} \mathcal{M}_{\lambda}, Q_{2}=\oplus_{\lambda \leq \lambda^{\prime}} \mathcal{M}_{\lambda}$ and take $\theta: Q_{2} \rightarrow Q_{1}$ to be the natural map with 
$\operatorname{coker} \theta \cong \mathcal{M}$. Take $Q^{\bullet}$ to be the cone of $\theta$ in $\mathrm{D}_{\mathrm{QCoh}}(X)$, for all integers $r$ we obtain an exact sequence in $\operatorname{Fun}_{S}^{\times}$:

$$
\mathbb{H}_{Q_{1}, \mathfrak{N} \bullet[r-1]} \longrightarrow \mathbb{H}_{\mathcal{Q}_{2}, \mathcal{N} \bullet[r-1]} \longrightarrow \mathbb{H}_{Q^{\bullet}, \mathfrak{N} \bullet[r]} \longrightarrow \mathbb{H}_{Q_{1}, \mathcal{N} \bullet[r]} \longrightarrow \mathbb{H}_{\mathcal{Q}_{2}, \mathfrak{N} \bullet[r]}
$$

For all integers $r$ we also have isomorphisms

$$
\mathbb{H}_{\mathcal{Q}_{1}, \mathcal{N} \cdot[r]} \cong \prod_{\lambda} \mathbb{H}_{\mathcal{M}_{\lambda}, \mathcal{N} \bullet[r]} \text { and } \quad \mathbb{H}_{\mathcal{Q}_{2}, \mathcal{N} \cdot[r]} \cong \prod_{\lambda \leq \lambda^{\prime}} \mathbb{H}_{\mathcal{M}_{\lambda}, \mathcal{N} \bullet[r]}
$$

By Examples 3.9 and 3.5, together with our hypotheses, we deduce that $\mathbb{H}_{Q^{\bullet}, \mathcal{N}^{\bullet}[r]}$ is a coherent functor for all $r \leq n$. Now, there is a distinguished triangle $\mathbb{Q}^{\bullet} \rightarrow$ $\mathcal{M}[-1] \rightarrow(\operatorname{ker} \theta)[1]$, thus we obtain an exact sequence in $\mathbf{F u n}_{S}^{\times}$for all integers $r$ :

$\mathbb{H}_{Q^{\bullet}, \mathcal{N}^{\bullet}[r-1]} \longrightarrow \mathbb{H}_{(\operatorname{ker} \theta), \mathcal{N}^{\bullet}[r-2]} \longrightarrow \mathbb{H}_{\mathcal{M}, \mathcal{N}^{\bullet}[r]} \longrightarrow \mathbb{H}_{Q^{\bullet}, \mathcal{N}^{\bullet}[r]} \longrightarrow \mathbb{H}_{(\operatorname{ker} \theta), \mathcal{N}^{\bullet}[r-1]}$

By hypothesis, $\mathbb{H}_{(\operatorname{ker} \theta), \mathcal{N} \bullet[r]}$ is coherent for all $r<n$. Taking $r=n$ in the exact sequence above and applying Example 3.5, we deduce that $\mathbb{H}_{\mathcal{M}, \mathcal{N}^{\bullet}[n]}$ is coherent.

Our next result forms the second part of the induction process. We wish to emphasize that in the following lemma, some of the pullbacks are underived. This is not a typographical error and is essential to the argument.

Lemma 4.5. Fix a 2-cartesian diagram of algebraic stacks:

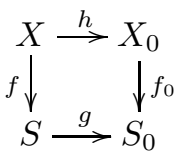

where $S$ and $S_{0}$ are affine schemes. Fix an integer $n$ and let $\mathcal{M}_{0}, \mathcal{N}_{0} \in \mathbf{Q C o h}\left(X_{0}\right)$. Assume, in addition, that $\mathcal{N}_{0}$ is of finite presentation, flat over $S_{0}$, and that the functors:

$$
\mathbb{H}_{\mathcal{M}_{0}, \mathcal{N}_{0}[l]} \text { and } \quad \mathbb{H}_{\mathcal{F},\left(h * \mathcal{N}_{0}\right)[r]}
$$

are coherent for all integers $l$ and for all integers $r<n$ and all $\mathcal{F} \in \mathbf{Q C o h}(X)$. Then, the functors $\mathbb{H}_{\left(h^{*} \mathcal{M}_{0}\right),\left(h^{*} \mathcal{N}_{0}\right)[r]}$ are coherent for all integers $r \leq n$.

Proof. Set $\mathcal{M} \cdot:=\operatorname{L} h_{\mathrm{Q}}^{*} \mathcal{M}_{0}$ and $\mathcal{N}:=h^{*} \mathcal{N}_{0}$. Given integers $r$ and $l$ set:

$$
V_{r, l}:=\mathbb{H}_{\tau \geq-l} \mathcal{M} \cdot, \mathcal{N}[r+l] \quad \text { and } \quad W_{r, l}:=\mathbb{H}_{\mathcal{H}^{-l}(\mathcal{M} \bullet} \cdot \mathcal{N} \mathfrak{N}[r] .
$$

It is sufficient to prove that $V_{r, l}$ is coherent for all $r \leq n$ and all $l$ (the result follows by taking $l=0)$. Now, for integers $r$ and $l$ we have a distinguished triangle in $\mathrm{D}_{\mathrm{QCoh}}(X)$ :

$$
\mathcal{H}^{-l-1}(\mathcal{M} \cdot)[l+1] \longrightarrow \tau^{\geq-l-1} \mathcal{M} \cdot \longrightarrow \tau^{\geq-l} \mathcal{M} \cdot
$$

Applying to this triangle the functor $\operatorname{Hom}_{\mathcal{O}_{X}}\left(-, \mathcal{N}[r+l] \otimes_{\mathcal{O}_{X}}^{\llcorner} L f_{\mathrm{Q}}^{*}(-)\right)$ we obtain an exact sequence in $\mathbf{F u n}_{S}^{\times}$:

$$
V_{r-2, l+1} \longrightarrow W_{r-2, l+1} \longrightarrow V_{r, l} \longrightarrow V_{r-1, l+1} \longrightarrow W_{r-1, l+1}
$$

By hypothesis, $W_{r, l}$ is coherent for all $l$ and all $r<n$. Thus, by Example 3.5 the result will follow from the assertion that $V_{r, l}$ is coherent for all $l$ and all $r<n$. By induction on $r$, the exact sequence (4.1) shows that it is sufficient to prove that $V_{r, l}$ is coherent for all integers $l$ and all $r \leq 0$. 


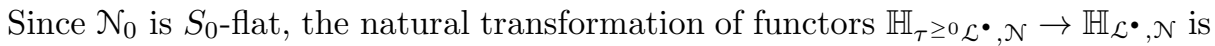
an isomorphism for all $\mathcal{L}^{\bullet} \in \mathrm{D}_{\mathrm{QCoh}}(X)$. Hence, if $l \geq 0$ and $r \leq 0$ :

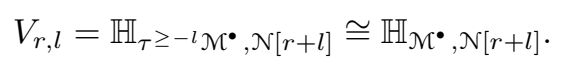

Moreover, if $l<0$, then $\tau^{\geq-l} \mathcal{M} \cdot \simeq 0$, so $V_{r, l} \equiv 0$. Thus, it remains to show that $\mathbb{H}_{\mathcal{M}} \cdot, \mathcal{N}[l]$ is coherent for all $l$. Let $I \in \mathbf{Q C o h}(S)$, then Lemma 2.2 gives a natural isomorphism $\mathbb{H}_{\mathcal{N}^{\bullet}, \mathcal{N}[l]}(I) \cong \mathbb{H}_{\mathcal{M}_{0}, \mathcal{N}_{0}[l]}\left(g_{*} I\right)$. Example 3.6 now gives the result.

We can now prove Theorem $\mathrm{C}$

Proof of Theorem $\square$. We must prove that $\mathrm{D}_{\mathrm{QCoh}}(X)=\mathcal{T}_{X / S}^{\mathcal{N}[0]}$. By Lemma 4.3, we may immediately reduce to the case where $f: X \rightarrow S$ is proper and of finite presentation. Next, by Lemma 4.2(2), it suffices to prove that $\mathbb{H}_{\mathcal{M}, \mathcal{N}[n]}$ is coherent for all integers $n$, and all quasicoherent $\mathcal{O}_{X}$-modules $\mathcal{M}$.

Now, $\mathbb{H}_{\mathcal{M}, \mathcal{N}[n]} \equiv 0$ for all $n<0$ and all $\mathcal{M} \in \mathbf{Q C o h}(X)$. We now prove, by induction on $n \geq-1$, that $\mathbb{H}_{\mathcal{M}, \mathcal{N}[n]}$ is coherent. Certainly, the result is true for $n=-1$. Thus, we fix $n \geq 0$ and assume the result has been proven for all $r<n$.

By Lemma 4.4 it suffices to prove that $\mathbb{H}_{\mathcal{M}, \mathcal{N}[n]}$ is coherent when $\mathcal{M}$ is of finite presentation. Thus, by standard limit methods [Ryd09, Prop. B.3], there exists an affine scheme $S_{0}$ of finite type over $\operatorname{Spec} \mathbb{Z}$, a proper morphism of algebraic stacks $f_{0}: X_{0} \rightarrow S_{0}$, and a morphism of affine schemes $g: S \rightarrow S_{0}$ inducing an isomorphism of algebraic stacks $h: X \rightarrow X_{0} \times_{S_{0}} S$. This data may be chosen so that there exists coherent $\mathcal{O}_{X_{0}}$-modules $\mathcal{M}_{0}$ and $\mathcal{N}_{0}$, with $\mathcal{N}_{0}$ flat over $S$, as well as isomorphisms of $\mathcal{O}_{X}$-modules $h^{*} \mathcal{N}_{0} \cong \mathcal{N}, h^{*} \mathcal{N}_{0} \cong \mathcal{M}$. By Theorem B and Example 3.13 the functors $\mathbb{H}_{\mathcal{M}_{0}, \mathcal{N}_{0}[l]}$ are coherent for all $l$. By Lemma 4.5 and the inductive hypothesis, the result follows.

\section{COHEREnCE OF Hom-FunCtors: NON-FLAT CASE}

In this section we prove Theorem E For this section we also retain the notation of 4 . We begin by dispatching Theorem $\mathrm{E}$ in the projective case.

Lemma 5.1. Fix an affine and noetherian scheme $S$, a morphism of schemes $f: X \rightarrow S$ which is projective, and $\mathcal{N}^{\bullet} \in \mathrm{D}_{\text {Coh }}^{b}(X)$. Then, $\mathcal{T}_{X / S}^{\mathcal{N}^{\bullet}}=\mathrm{D}_{\mathrm{QCoh}}(X)$.

Proof. By Lemma 4.2(1), it is sufficient to show that $\mathrm{D}_{\mathrm{QCoh}}^{-}(X) \subseteq \mathcal{T}_{X / S}^{\mathcal{N}^{\bullet}}$. Since $f$ is a projective morphism and $S$ is an affine and noetherian scheme, $f$ has an ample family of line bundles. It now follows from [SGA6, II.2.2.9] that if $\mathcal{M}^{*} \in \mathrm{D}_{\mathrm{QCoh}}^{-}(X)$, then $\mathcal{M}^{\bullet}$ is quasi-isomorphic to a complex $Q^{\bullet}$ whose terms are direct sums of shifts of line bundles. Thus, by Lemma 4.2 it is sufficient to prove that if $\mathcal{L} \in \mathbf{C o h}(X)$ is a line bundle, then $\mathcal{L}[0] \in \mathcal{T}_{X / S}^{\mathcal{N}^{*}}$. Fix $n \in \mathbb{Z}$, then we have natural isomorphisms:

$$
\begin{aligned}
\mathbb{H}_{\mathcal{L}[0], \mathcal{N}^{\bullet}[n]} & :=\operatorname{Hom}_{\mathcal{O}_{X}}\left(\mathcal{L}[0], \mathcal{N}^{\bullet}[n] \otimes_{\mathcal{O}_{X}}^{\mathrm{L}} \mathrm{L} f_{\mathrm{Q}^{*}}^{*}(-)\right) \\
& \cong \mathcal{H}^{0}\left(\operatorname { R } \Gamma \left(X,\left(\mathcal{L}^{-1}[0] \otimes_{\mathcal{O}_{X}}^{\mathrm{L}} \mathcal{N}^{\bullet}[n] \otimes_{\mathcal{O}_{X}}^{\mathrm{L}} \mathrm{L} f_{\mathrm{Q}}^{*}(-)\right)\right.\right. \\
& \cong \mathcal{H}^{0}\left(\operatorname{R} \Gamma\left(X, \mathcal{L}^{-1}[0] \otimes_{\mathcal{O}_{X}}^{\mathrm{L}} \mathcal{N}^{\bullet}[n]\right) \otimes_{\mathcal{O}_{S}}^{\mathrm{L}}(-)\right),
\end{aligned}
$$

the latter isomorphism is given by the projection formula (1.6). Since $\mathcal{L}$ is $\mathcal{O}_{X^{-}}$ flat and $\mathcal{N}^{\bullet} \in \mathrm{D}_{\text {Coh }}^{b}(X)$, it follows that $\mathcal{L}^{-1}[0] \otimes_{\mathcal{O}_{X}}^{\mathrm{L}} \mathcal{N}^{\bullet}[n] \in \mathrm{D}_{\text {Coh }}^{b}(X)$. Hence, $\mathrm{R} \Gamma\left(X, \mathcal{L}^{-1}[0] \otimes_{\mathcal{O}_{X}}^{\mathrm{L}} \mathcal{N}^{\bullet}[n]\right) \in \mathrm{D}_{\mathrm{Coh}}^{b}(S)$ [SGA6, III.2.2]. By Example 3.13, $\mathbb{H}_{\mathcal{L}, \mathfrak{N}^{\bullet}[n]}$ is coherent, so $\mathcal{L}[0] \in \mathcal{T}_{X / S}^{\mathcal{N}^{*}}$. 
We can now prove Theorem E

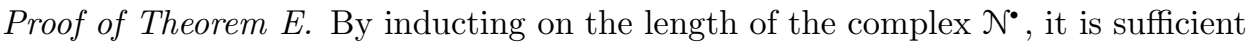
to prove the result when $\mathcal{N}^{\bullet}$ is only supported in cohomological degree 0 . In particular, there exists a closed immersion $i: Y \rightarrow X$, such that the composition $f \circ i$ is proper, together with a coherent $\mathcal{O}_{Y}$-module $\mathcal{N}_{0}$ and a quasi-isomorphism $i_{*} \mathcal{N}_{0}[0] \cong \mathcal{N}^{*}$. By Lemma 4.3 , it suffices to prove that $\mathcal{T}_{Y / S}^{\mathcal{N}_{0}[0]}=\mathrm{D}_{\mathrm{QCoh}}(Y)$. Hence, we have reduced the claim to the case where the morphism $f$ is proper and where $\mathcal{N}^{\bullet} \simeq \mathcal{N}[0]$ for some $\mathcal{N} \in \mathbf{C o h}(X)$.

Now let $\mathcal{C}_{X / S} \subset \operatorname{Coh}(X)$ denote the full subcategory with objects those $\mathcal{N} \in$ $\operatorname{Coh}(X)$ such that $\mathcal{T}_{X / S}^{\mathcal{N}[0]}=\mathrm{D}_{\mathrm{QCoh}}(X)$. By the 5 -Lemma, it is plain to see that $\mathcal{C}_{X / S}$ is an exact subcategory (in the sense of [EGA, III.3.1]). We now prove by noetherian induction on the closed substacks of $X$ that $\mathcal{C}_{X / S}=\mathbf{C o h}(X)$. By virtue of Lemma 4.3 and the technique of dévissage EGA, Proof of III.3.2], it is sufficient to prove that $\mathcal{C}_{X / S}=\operatorname{Coh}(X)$ when $X$ is integral and $\mathcal{T}_{X / S}^{\mathrm{Q}[0]}=\mathrm{D}_{\mathrm{QCoh}}(X)$ for all coherent $\mathcal{O}_{X}$-modules $\mathcal{Q}$ such that supp $\mathcal{Q} \subsetneq|X|$.

So, we fix $\mathcal{N} \in \operatorname{Coh}(X)$. Combining Chow's Lemma [EGA, II.5.6.1] with [EHKV01, Thm. 2.7], there exists a morphism $p: X^{\prime} \rightarrow X$ that is proper, surjective, and generically finite such that $X^{\prime}$ is a projective $S$-scheme. The diagonal of $X$ is finite, thus $X^{\prime \prime}:=X^{\prime} \times_{X} X^{\prime}$ is also a projective $S$-scheme, denote by $q: X^{\prime \prime} \rightarrow X$ the induced morphism. By Lemma 5.1 we deduce that $\mathcal{T}_{X^{\prime} / S}^{p^{*} \mathcal{N}[0]}=\mathrm{D}_{\mathrm{QCoh}}\left(X^{\prime}\right)$ and $\mathcal{T}_{X^{\prime \prime} / S}^{q^{*} \mathcal{N}[0]}=\mathrm{D}_{\mathrm{QCoh}}\left(X^{\prime \prime}\right)$.

Next, by Lemma 4.3. $\mathcal{T}_{X / S}^{\mathrm{R} p * p^{*} \mathcal{N}}=\mathrm{D}_{\mathrm{QCoh}}(X)$. Also, $p$ is generically finite, thus generically affine, so the support of the cohomology sheaves of $\tau^{\geq 1}\left(\operatorname{R} p_{*} p^{*} \mathcal{N}\right)$ vanishes generically. In particular, by noetherian induction, we deduce that $\mathcal{T}_{X / S}^{\tau^{\geq 1}}\left(\mathrm{R} p_{*} p^{*} \mathcal{N}\right)=$ $\mathrm{D}_{\mathrm{QCoh}}(X)$, thus $\mathcal{T}_{X / S}^{p_{*} p^{*} \mathcal{N}[0]}=\mathrm{D}_{\mathrm{QCoh}}(X)$. An identical analysis for $q$ also proves that $\mathcal{T}_{X / S}^{q_{*} q^{*} \mathcal{N}[0]}=\mathrm{D}_{\mathrm{QCoh}}(X)$ and so $p_{*} p^{*} \mathcal{N}$ and $q_{*} q^{*} \mathcal{N} \in \mathcal{C}_{X / S}$. Hence, the equalizer $\tilde{\mathcal{N}}$ of the two maps $p_{*} p^{*} \mathcal{N} \rightrightarrows q_{*} q^{*} \mathcal{N}$ belongs to $\mathcal{C}_{X / S}$. Of course, there is also a natural map $\theta: \mathcal{N} \rightarrow \tilde{\mathcal{N}}$. But $X$ is integral, thus there is a dense open $U \subset X$ such that $p^{-1}(U) \rightarrow U$ is flat. By flat descent, $\theta$ is an isomorphism over $U$. So the exactness of the subcategory $\mathcal{C}_{X / S} \subset \mathbf{C o h}(X)$ and dévissage now prove that $\mathcal{N} \in \mathcal{C}_{X / S}$.

\section{Applichtions}

6.1. Representability of Hom-spaces. As promised, Theorem $\mathrm{D}$ is now completely elementary.

Proof of Theorem $D$, The latter claim follows from the former by standard limit methods. Now, $\underline{\operatorname{Hom}}_{\mathcal{O}_{X} / S}(\mathcal{N}, \mathcal{N})$ is an étale sheaf, thus it is sufficient to prove the result in the case where $S$ is affine. By Theorem $[$, the functor:

$$
\operatorname{Hom}_{\mathcal{O}_{X}}\left(\mathcal{M}, \mathcal{N} \otimes_{\mathcal{O}_{X}} f^{*}(-)\right): \mathbf{Q C o h}(S) \rightarrow \mathbf{A b}
$$

is coherent. The flatness of $\mathcal{N}$ over $S$ also shows that this functor is left-exact, so by Example 3.10 , it is corepresentable by a quasicoherent $\mathcal{O}_{S}$-module $Q_{\mathcal{N}, \mathcal{N}}$. So, 
fixing an affine $S$-scheme $(T \stackrel{\tau}{\rightarrow} S)$, there are natural isomorphisms:

$$
\begin{aligned}
& \underline{\operatorname{Hom}}_{\mathcal{O}_{X} / S}(\mathcal{M}, \mathcal{N})[T \stackrel{\tau}{\rightarrow} S]=\operatorname{Hom}_{\mathcal{O}_{X}}\left(\tau_{X}^{*} \mathcal{M}, \tau_{X}^{*} \mathcal{N}\right) \cong \operatorname{Hom}_{\mathcal{O}_{X}}\left(\mathcal{M},\left(\tau_{X}\right)_{*} \tau_{X}^{*} \mathcal{N}\right) \\
& \cong \operatorname{Hom}_{\mathcal{O}_{X}}\left(\mathcal{M}, \mathcal{N} \otimes_{\mathcal{O}_{X}} f^{*}\left[\tau_{*} \mathcal{O}_{T}\right]\right) \\
& \cong \operatorname{Hom}_{\mathcal{O} S}\left(Q_{\mathcal{N}, \mathcal{N}}, \tau_{*} \mathcal{O}_{T}\right) \\
& \cong \operatorname{Hom}_{\mathcal{O}_{S}-\mathrm{Alg}}\left(\operatorname{Sym}_{\mathcal{O}_{S}} Q_{\mathcal{N}, \mathcal{N}}, \tau_{*} \mathcal{O}_{T}\right) \\
& \cong \operatorname{Hom}_{\mathrm{Sch} / S}\left(T \stackrel{\tau}{\rightarrow} S, \underline{\operatorname{Spec}}_{\mathcal{O}_{S}} \operatorname{Sym}_{\mathcal{O}_{S}} Q_{\mathcal{M}, \mathcal{N}}\right) \text {. }
\end{aligned}
$$

The natural isomorphism above extends to all $S$-schemes as $\underline{\operatorname{Hom}}_{\mathcal{O}_{X} / S}(\mathcal{N}, \mathcal{N})$ is an étale sheaf. The result follows.

6.2. Cohomology and base change. To prove Theorem $\mathrm{A}$, we will consider some refinements of the vanishing results of A. Ogus and G. Bergman OB72 that occur in the setting of finitely generated functors. So, we fix a ring $A$ and an $A$-linear functor $Q: \operatorname{Mod}(A) \rightarrow \operatorname{Mod}(A)$. Define

$$
\mathbb{V}(Q)=\left\{\mathfrak{p} \in \operatorname{Spec} A: Q(N)=0 \quad \forall N \in \operatorname{Mod}\left(A_{\mathfrak{p}}\right)\right\} .
$$

Finitely generated functors immediately demonstrate their utility.

Proposition 6.1. Fix a ring $A$ and an $A$-linear functor $F: \operatorname{Mod}(A) \rightarrow \operatorname{Mod}(A)$ which preserves direct limits. If the functor $F$ is finitely generated, then the set $\mathbb{V}(F) \subset \operatorname{Spec} A$ is Zariski open.

Proof. Fix a generator $(I, \eta)$ for $F$ and a prime ideal $\mathfrak{p} \triangleleft A$ such that $F_{A_{\mathfrak{p}}} \equiv 0$. For $a \in A$ there is the localisation morphism $l_{a}: I \rightarrow I_{a}$ (resp. $\left.l_{\mathfrak{p}}: I \rightarrow I_{\mathfrak{p}}\right)$ and we set $\eta_{a}=\left(l_{a}\right)_{*} \eta\left(\right.$ resp. $\left.\eta_{\mathfrak{p}}=\left(l_{\mathfrak{p}}\right)_{*} \eta\right)$. Since $F\left(I_{\mathfrak{p}}\right)=0$, it follows that $\eta_{\mathfrak{p}}=0$. However, as $I_{\mathfrak{p}}=\lim _{a \notin \mathfrak{p}} I_{a}$ and the functor $F$ commutes with direct limits of $A$-modules, there exists $a \notin \mathfrak{p}$ such that $\eta_{a}=0$ in $F\left(I_{a}\right)$. Since the pair $\left(I_{a}, \eta_{a}\right)$ generates $F_{A_{a}}$, we have that $F_{A_{a}} \equiv 0$.

We now record for future reference a result that is likely well-known, though we are unaware of a reference. For an $A$-module $N$, define $Q^{N}: \operatorname{Mod}(A) \rightarrow$ Sets to be the functor $Q\left(-\otimes_{A} N\right): \operatorname{Mod}(A) \rightarrow$ Sets. For another ring $C$, a $C$-module $K$, and a functor $G: \operatorname{Mod}(A) \rightarrow \operatorname{Mod}(C)$, there is the functor $G \otimes_{C} K: \operatorname{Mod}(A) \rightarrow$ $\operatorname{Mod}(C): M \mapsto G(M) \otimes_{C} K$. Given an $A$-linear functor $H: \operatorname{Mod}(A) \rightarrow \operatorname{Mod}(A)$, and any $A$-module $N$, there is a natural transformation of functors

$$
\delta_{H, N}: H \otimes_{A} N \Rightarrow H^{N} .
$$

Proposition 6.2. Fix a ring $A$, and an A-linear functor $F: \operatorname{Mod}(A) \rightarrow \operatorname{Mod}(A)$. Suppose that the functor $F$ preserves direct limits. Then, for any flat $A$-module $M$, the natural transformation

$$
\delta_{F, M}: F \otimes_{A} M \Rightarrow F^{M}
$$

is an isomorphism of functors.

Proof. First, assume that the $A$-module $M$ is finite free. The functor $F$ commutes with finite products, thus the natural transformation $\delta_{F, M}$ induces an isomorphism. For the general case, by Lazard's Theorem [Laz64], we may write $M=\lim _{i} P_{i}$, where each $P_{i}$ is a finite free $A$-module. Since tensor products commute with direct limits, as does the functor $F$, the Proposition follows from the case already considered. 
Corollary 6.3. Fix a noetherian ring $R$ and a bounded $R$-linear functor $G$ : $\operatorname{Mod}(R) \rightarrow \operatorname{Mod}(R)$ that commutes with direct limits.

(1) For any quasi-finite $R$-algebra $R^{\prime}$, the functor $G_{R^{\prime}}$ is bounded.

(2) For any $\mathfrak{p} \in \operatorname{Spec} R$, the functor $G_{R_{\mathfrak{p}}}$ is bounded.

Proof. For (1), by Zariski's Main Theorem [EGA, IV.18.12.13], the homomorphism $R \rightarrow R^{\prime}$ factors as $R \rightarrow \widetilde{R} \rightarrow R^{\prime}$ where $R \rightarrow \widetilde{R}$ is finite and Spec $R^{\prime} \rightarrow \operatorname{Spec} \widetilde{R}$ is an open immersion. Since the functor $G$ is bounded, the functor $G_{\widetilde{R}}$ is bounded. Thus, it suffices to consider the case where $\operatorname{Spec} R^{\prime} \rightarrow \operatorname{Spec} R$ is an open immersion. For any coherent $R^{\prime}$-module $M^{\prime}$, there exists a coherent $R$-module $M$ together with an isomorphism of $R^{\prime}$-modules $M \otimes_{R} R^{\prime} \cong M^{\prime}$. Since the homomorphism $R \rightarrow R^{\prime}$ is flat, Proposition 6.2 implies that $G(M) \otimes_{R} R^{\prime} \cong G\left(M \otimes_{R} R^{\prime}\right) \cong G\left(M^{\prime}\right)$. The functor $G$ is bounded, thus the $R^{\prime}$-module $G(M) \otimes_{R} R^{\prime}$ is coherent, giving the claim.

For (2), fix a coherent $R_{\mathfrak{p}}$-module $N$, then for some $f \in R-\mathfrak{p}$ there exists an $R_{f^{-}}$ module $L$, together with an $R_{\mathfrak{p}}$-module isomorphism $L \otimes_{R_{f}} R_{\mathfrak{p}} \cong N$. By Proposition 6.2, we have $G(N) \cong G(L) \otimes_{R_{f}} R_{\mathfrak{p}}$. By (1), since $R \rightarrow R_{f}$ is quasi-finite, it follows that $G_{R_{f}}$ is bounded. Thus, $G(N)$ is a coherent $R_{\mathfrak{p}}$-module.

Remark 6.4. Corollary 6.3(2) also holds for the henselization and strict henselization of $R_{\mathfrak{p}}$.

Fix a noetherian ring $R$ and a half-exact, bounded, $R$-linear functor $F: \operatorname{Coh}(R) \rightarrow$ $\operatorname{Coh}(R)$. A. Ogus and G. Bergman show in OB72, Thm. 2.1] that if for all closed points $\mathfrak{q} \in \operatorname{Spec} R$ we have $F(\kappa(\mathfrak{q}))=0$, then $F$ is the zero functor. We have the following amplification.

Corollary 6.5. Fix a noetherian ring $R$ and a bounded, half-exact, $R$-linear functor $G: \operatorname{Mod}(R) \rightarrow \operatorname{Mod}(R)$ which commutes with direct limits. Then,

$$
\mathbb{V}(G)=\{\mathfrak{q} \in \operatorname{Spec} R: G(\kappa(\mathfrak{q}))=0\} .
$$

Proof. Clearly, if $\mathfrak{q} \in \mathbb{V}(G)$, then $G(\kappa(\mathfrak{q}))=0$. For the other inclusion, let $\mathfrak{q} \in$ Spec $R$ satisfy $G(\kappa(\mathfrak{q}))=0$. By Corollary $6.3(2)$, the functor $G_{R_{\mathfrak{q}}}$ is bounded. Thus, OB72, Thm. 2.1] applies, giving $G_{R_{\mathfrak{q}}} \equiv 0$, and so $\mathfrak{q} \in \mathbb{V}(G)$.

An $R$-linear functor $G: \operatorname{Mod}(R) \rightarrow \operatorname{Mod}(R)$ is universally bounded if for any noetherian $R$-algebra $R^{\prime}$, the functor $G_{R^{\prime}}: \operatorname{Mod}\left(R^{\prime}\right) \rightarrow \operatorname{Mod}\left(R^{\prime}\right)$ is bounded. To combine Proposition 6.1 and Corollary [6.5] it is useful to have the following easily proven Lemma at hand.

Lemma 6.6. Fix a ring $A$ and an $A$-linear functor $F: \operatorname{Mod}(A) \rightarrow \operatorname{Mod}(A)$ preserving direct limits. If the functor $F$ is finitely generated, then there exists a generator $(I, \eta)$ with $I$ a finitely presented $A$-module. In particular, if the ring $A$ is noetherian, then the functor $F$ is universally bounded.

Combining Proposition 6.1. Corollary 6.5, and Lemma 6.6, we obtain the vanishing result we desire.

Corollary 6.7. Fix a noetherian ring $R$ and an $R$-linear, half-exact functor $F$ : $\operatorname{Mod}(R) \rightarrow \operatorname{Mod}(R)$ which is finitely generated and preserves direct limits. If $\mathfrak{q} \in \operatorname{Spec} R$ and $F(\kappa(\mathfrak{q}))=0$, then there exists $r \in R-\mathfrak{q}$ such that $F_{R_{r}} \equiv 0$.

Proof. By Corollary6.5, $\mathfrak{q} \in \mathbb{V}(F)$. By Proposition 6.1, the set $\mathbb{V}(F)$ is Zariski open, thus there exists $r \in R-\mathfrak{q}$ such that $\mathfrak{p} \in \operatorname{Spec} R_{r} \subset \mathbb{V}(F)$. Let $N \in \operatorname{Mod}\left(R_{r}\right)$ 
and $\mathfrak{p} \in \operatorname{Spec} R_{r}$, then by Proposition 6.2 it follows that $F(N)_{\mathfrak{p}}=F\left(N_{\mathfrak{p}}\right)$. But $\mathfrak{p} \in \mathbb{V}(F)$ and so $F(N)_{\mathfrak{p}}=0$. Since $F(N)$ is an $R_{r}$-module, the result follows.

We now combine Corollary 6.7 with the exchange property proved by A. Ogus and G. Bergman [OB72, Cor. 5.1]. Some notation: for a ring $A$, a pair of $A$-linear functors $F_{0}, F_{1}: \operatorname{Mod}(A) \rightarrow \operatorname{Mod}(A)$ is cohomological if for any exact sequence of $A$-modules $0 \rightarrow M^{\prime} \rightarrow M \rightarrow M^{\prime \prime} \rightarrow 0$, there is a functorially induced exact sequence of $A$-modules:

$$
F_{0}\left(M^{\prime}\right) \longrightarrow F_{0}(M) \longrightarrow F_{0}\left(M^{\prime \prime}\right) \longrightarrow F_{1}\left(M^{\prime}\right) \longrightarrow F_{1}(M) \longrightarrow F_{1}\left(M^{\prime \prime}\right) .
$$

Corollary 6.8 (Property of exchange). Fix a noetherian ring $R$ and a cohomological pair of $R$-linear functors $F_{0}, F_{1}: \operatorname{Mod}(R) \rightarrow \operatorname{Mod}(R)$ which are finitely generated and preserve direct limits. For $i=0,1$ and for any $M \in \operatorname{Mod}(R)$, there is a natural map:

$$
\phi_{i}(M): F_{i}(R) \otimes_{R} M \rightarrow F_{i}(M) .
$$

Let $\mathfrak{q} \in \operatorname{Spec} R$ and suppose that $\phi_{0}(\kappa(\mathfrak{q}))$ is surjective. Then,

(1) there exists $r \in R-\mathfrak{q}$ such that for all $M \in \mathbf{Q} \mathbf{C o h}\left(R_{r}\right)$, the map $\phi_{0}(M)$ is an isomorphism.

(2) The following are equivalent:

(a) $\phi_{1}(\kappa(\mathfrak{q}))$ is surjective;

(b) the $R_{\mathfrak{q}}$-module $F_{1}(R)_{\mathfrak{q}}$ is free.

Proof. By Lemma 6.6, the functors $F_{i}$ are bounded. Now apply [OB72, Cor. 5.1] and Corollary 6.7.

Proof of Theorem $\AA$. Throughout we fix a 2-cartesian diagram of noetherian algebraic stacks:

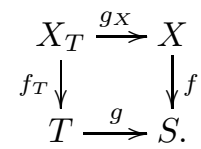

The results are all smooth local on $S$ and $T$, thus we may assume that $S=\operatorname{Spec} A$ and $T=\operatorname{Spec} B$ and $g$ is induced by a ring homomorphism $A \rightarrow B$. We may also work with the global Ext-groups instead of the relative $\mathcal{E} x t$-sheaves.

For an $A$-module $I$ set $E^{q}(I)=\operatorname{Ext}_{\mathcal{O}_{X}}^{q}\left(\mathcal{M}^{\bullet}, \mathcal{N} \otimes_{\mathcal{O}_{X}} f^{*} I\right)$, which gives an $A$-linear functor $\operatorname{Mod}(A) \rightarrow \operatorname{Mod}(A)$. By Theorem $\mathbb{C}$, the functor $E^{q}$ is coherent and by Lemma 1.1 the functor preserves filtered colimits (in particular, it preserves direct limits). By Lemma 2.2, if $J$ is a $B$-module, there is a natural isomorphism:

$$
E^{q}(J)=\operatorname{Ext}_{\mathcal{O}_{X}}^{q}\left(\mathcal{M} \cdot, \mathcal{N} \otimes_{\mathcal{O}_{X}} f^{*} g_{*} J\right) \cong \operatorname{Ext}_{\mathcal{O}_{X_{T}}}^{q}\left(\mathrm{~L}\left(g_{X}\right)_{\mathrm{Q}}^{*} \mathcal{M} \mathfrak{C}^{*}, g_{X}^{*} \mathcal{N} \otimes_{\mathcal{O}_{X_{T}}} f_{T}^{*} J\right) .
$$

In particular, taking $J=B$ we obtain a natural map:

$$
E^{q}(A) \otimes_{A} B:=\operatorname{Ext}_{\mathcal{O}_{X}}^{q}(\mathcal{M} \cdot, \mathcal{N}) \otimes_{A} B \rightarrow \operatorname{Ext}_{\mathcal{O}_{X_{T}}}^{q}\left(\mathrm{~L}\left(g_{X}\right)_{\mathrm{Q}}^{*} \mathcal{M} \cdot, g_{X}^{*} \mathcal{N}\right)=E^{q}(B) .
$$

The result now follows from Corollary 6.8 . 


\section{REFERENCES}

[AK80] A. B. Altman and S. L. Kleiman, Compactifying the Picard scheme, Adv. in Math. 35 (1980), no. 1, 50-112.

[Aus66] M. Auslander, Coherent functors, Proc. Conf. Categorical Algebra (La Jolla, Calif., 1965), Springer, New York, 1966, pp. 189-231.

[Beh03] K. Behrend, Derived l-adic categories for algebraic stacks, Mem. Amer. Math. Soc. 163 (2003), no. 774, viii+93.

[BF97] K. Behrend and B. Fantechi, The intrinsic normal cone, Invent. Math. 128 (1997), no. $1,45-88$.

[Bro12] S. Brochard, Finiteness theorems for the Picard objects of an algebraic stack, Adv. Math. 229 (2012), no. 3, 1555-1585.

[EGA] A. Grothendieck, Éléments de géométrie algébrique, I.H.E.S. Publ. Math. 4, 8, 11, 17, 20, 24, 28, 32 (1960, 1961, 1961, 1963, 1964, 1965, 1966, 1967).

[EHKV01] D. Edidin, B. Hassett, A. Kresch, and A. Vistoli, Brauer groups and quotient stacks, Amer. J. Math. 123 (2001), no. 4, 761-777.

$\left[\mathrm{FGI}^{+} 05\right] \quad$ B. Fantechi, L. Göttsche, L. Illusie, S. L. Kleiman, N. Nitsure, and A. Vistoli, Fundamental algebraic geometry, Mathematical Surveys and Monographs, vol. 123, American Mathematical Society, Providence, RI, 2005, Grothendieck's FGA explained.

[Fle81] H. Flenner, Ein Kriterium für die Offenheit der Versalität, Math. Z. 178 (1981), no. 4, 449-473.

[Fle82] Eine Bemerkung über relative Ext-Garben, Math. Ann. 258 (1981/82), no. 2, $175-182$.

[Hal12] J. Hall, Openness of versality via coherent functors, ArXiv e-prints (2012), arXiv:1206.4182

[Har66] R. Hartshorne, Residues and duality, Lecture notes of a seminar on the work of A. Grothendieck, given at Harvard 1963/64. With an appendix by P. Deligne. Lecture Notes in Mathematics, No. 20, Springer-Verlag, Berlin, 1966.

[Har98] Coherent functors, Adv. Math. 140 (1998), no. 1, 44-94.

[HR12a] J. Hall and D. Rydh, Coherence criteria for half-exact functors, In preparation (2012).

[HR12b] - General Hilbert stacks and Quot schemes, In preparation (2012).

[HR12c] - Tame stacks are perfect, In preparation (2012).

[Kra03] H. Krause, Coherent functors and covariantly finite subcategories, Algebr. Represent. Theory 6 (2003), no. 5, 475-499.

[KS06] M. Kashiwara and P. Schapira, Categories and sheaves, Grundlehren der Mathematischen Wissenschaften [Fundamental Principles of Mathematical Sciences], vol. 332, Springer-Verlag, Berlin, 2006.

[Laz64] D. Lazard, Sur les modules plats, C. R. Acad. Sci. Paris 258 (1964), 6313-6316.

[Lie06] M. Lieblich, Remarks on the stack of coherent algebras, Int. Math. Res. Not. (2006), Art. ID 75273, 12.

[LMB] G. Laumon and L. Moret-Bailly, Champs algébriques, Ergebnisse der Mathematik und ihrer Grenzgebiete. 3. Folge., vol. 39, Springer-Verlag, Berlin, 2000.

[LO08] Y. Laszlo and M. Olsson, The six operations for sheaves on Artin stacks. I. Finite coefficients, Publ. Math. Inst. Hautes Études Sci. (2008), no. 107, 109-168.

[Lur04] J. Lurie, Tannaka duality for geometric stacks, arXiv:math/0412266.

[Mum70] D. Mumford, Abelian varieties, Tata Institute of Fundamental Research Studies in Mathematics, No. 5, Published for the Tata Institute of Fundamental Research, Bombay, 1970 .

[Nee96] A. Neeman, The Grothendieck duality theorem via Bousfield's techniques and Brown representability, J. Amer. Math. Soc. 9 (1996), no. 1, 205-236.

[Nee11] A. Neeman, Non-left-complete derived categories, ArXiv e-prints (2011), arXiv:1103.5539

[OB72] A. Ogus and G. Bergman, Nakayama's lemma for half-exact functors, Proc. Amer. Math. Soc. 31 (1972), 67-74.

[Ols06] M. Olsson, $\underline{\text { Hom}}-$ stacks and restriction of scalars, Duke Math. J. 134 (2006), no. 1, 139-164.

[Ols07] _ Sheaves on Artin stacks, J. Reine Angew. Math. 603 (2007), 55-112. 
[RS09] M. Roth and J. M. Starr, A local-global principle for weak approximation on varieties over function fields, ArXiv e-prints (2009), arXiv:0908.0096

[Ryd09] D. Rydh, Noetherian approximation of algebraic spaces and stacks, ArXiv e-prints (2009), arXiv:0904.0227

[SGA6] Théorie des intersections et théorème de Riemann-Roch, Lecture Notes in Mathematics, Vol. 225, Springer-Verlag, Berlin, 1971, Séminaire de Géométrie Algébrique du Bois-Marie 1966-1967 (SGA 6), Dirigé par P. Berthelot, A. Grothendieck et L. Illusie. Avec la collaboration de D. Ferrand, J. P. Jouanolou, O. Jussila, S. Kleiman, M. Raynaud et J. P. Serre.

[Stacks] The Stacks Project Authors, Stacks Project, http://math.columbia.edu/algebraic_geometry/stacks-git

[Wat60] C. E. Watts, Intrinsic characterizations of some additive functors, Proc. Amer. Math. Soc. 11 (1960), 5-8.

Department of Mathematics, KTH, 10044 Stockholm, Sweden

E-mail address: jackhall@kth.se 